\title{
Numerical Solutions for Laminar Boundary Layer Nanofluid Flow along with a Moving Cylinder with Heat Generation, Thermal Radiation, and Slip Parameter
}

\author{
Titilayo Morenike Agbaje $(\mathbb{D}$ and Gilbert Makanda \\ Center for Sustainable Smart Cities, Department of Mathematical and Physical Sciences, Central University of Technology, \\ Free State, Private Bag X20539, Bloemfontein 9300, South Africa
}

Correspondence should be addressed to Titilayo Morenike Agbaje; titilayoagbaje@gmail.com

Received 15 April 2021; Accepted 5 November 2021; Published 1 December 2021

Academic Editor: Fasma Diele

Copyright ( $) 2021$ Titilayo Morenike Agbaje and Gilbert Makanda. This is an open access article distributed under the Creative Commons Attribution License, which permits unrestricted use, distribution, and reproduction in any medium, provided the original work is properly cited.

\begin{abstract}
The investigation of the numerical solution of the laminar boundary layer flow along with a moving cylinder with heat generation, thermal radiation, and surface slip effect is carried out. The fluid mathematical model developed from the Navier-Stokes equations resulted in a system of partial differential equations which were then solved by the multidomain bivariate spectral quasilinearization method (MD-BSQLM). The results show that increasing the velocity slip factor results in an enhanced increase in velocity and temperature profiles. Increasing the heat generation parameter increases temperature profiles; increasing the radiation parameter and the Eckert numbers both increase the temperature profiles. The concentration profiles decrease with increasing radial coordinate. Increasing the Brownian motion and the thermophoresis parameter both destabilizes the concentration profiles. Increasing the Schmidt number reduces temperature profiles. The effect of increasing selected parameters: the velocity slip, Brownian motion, and the radiation parameter on all residual errors show that these errors do not deteriorate. This shows that the MD-BSQLM is very accurate and robust. The method was compared with similar results in the literature and was found to be in excellent agreement.
\end{abstract}

\section{Introduction}

The boundary layer flow on heat and mass transfer has been overmoving, and stretching surfaces have been studied and remained an active area in the past decade. This is because it has numerous applications in areas such as hot rolling, processes of polymer extrusions, wire drawing, extrusions in aerodynamic plastic sheets, process of condensation in metallic plates during cooling, and many other applications. According to Poply et al. [1], the study of flows in cylinders is considered two-dimensional when the cylinder radius is much larger than the boundary layer thickness. For lean and thin cylinders, the two dimensions may be of the same order; in this case, the flow is referred to as axisymmetric rather than two-dimensional. These dimensions affect velocity, temperature, and concentration profiles which in turn affect the skin friction coefficient.
In light of the importance of laminar boundary layer fluid flow, many researchers have considered several flow geometries with different boundary conditions, using many different techniques to solve similar models. These include the research carried out by Shateyi and Marewo [2] who used the successive linearization method (SRM) to solve a problem on laminar boundary layer flow and heat transfer in nonlinear differential equations; they also considered stretching cylinder, porous media, and thermal conductivity. In their investigation, they observed that the curvature significantly affects temperature and velocity fields. Also, both the skin friction coefficient and local Nusselt number increase as the curvature increases. Rangi and Naseem [3] used the Keller-box technique to solve the equations describing boundary layer flow of heat transfer with nonconstant thermal conductivity along with a stretching cylinder. Their results also showed that the cylinder curvature affects 
temperature, velocity, and skin friction fields. In the results, thermal conductivity and curvature aid heat transfer and reduction in fluid viscosity aids the convectional heat transfer rate. Numerical solutions concerning boundary layer flow, heat transfer along a stretching cylinder in porous media were obtained by Mukhopadhyay [4] using the shooting method. The results of this study found that increasing permeability parameter results in a decrease in velocity profiles. The skin friction and rate of heat transfer are much less on the flat plate than on the cylinder surface. Some other laminar boundary layer fluid models by different researchers including Elbashbeshy et al. [5], Lin and Shih [6], and Ali and Alabdulkarem [7] among others investigated the Casson fluid flow on a stretching surface based on an exponential model; they also applied theoretical analysis using lie groups in MHD fluid flow. In these works, a fourth-order Runge-Kutta method was used.

The study of the fluid flow and the physical properties of nanofluids has been widely studied in the past few years due to the wide applications of these fluids in cancer therapy, fuel cells, electronics, and pharmaceutical processes, just to mention a few. The concept of nanofluids was introduced by Choi [8], where the introduction for the proposal of nanoparticles suspended in base fluids such as ethylene glycol, oil, and water. A nonhomogeneous equation with two components for nanofluids was introduced by Buongiorno [9], where the seven slip mechanisms were proposed. The results explained effects in thermophoresis Brownian motion in nanofluids. Alamri et al. [10] used the homotopy analysis method to investigate fluid flow in nanofluids in the presence of secondorder slip and Stefan blowing effects in a tube (Poiseuille). In this investigation, the results show that the retarding effects of Stefan blowing is observed for temperature and velocity profiles, the opposite effect was noticed for the case of particle concentrations. A more enhanced response in the field of velocity was observed in the slip of the second order than in the slip of the first order. Nadeem et al. [11] used the homotopy analysis method in the solutions for nonlinear differential equations in which the oblique stagnation point flow was considered for a Casson nanofluid flow with stretching surface and heat transfer. The Runge-Kutta method of the fourth order together with the shooting technique was used by Khan et al. [12] to find a numerical solution of the Maxwell nanofluid stagnation point fluid flow over a stretching sheet with slip conditions and chemical reaction effects. Their results showed that the skin friction coefficient is inversely proportional to slip parameter but an opposite is noticed in the case of fluid relaxation parameter.

Dhanai et al. [13] investigated MHD boundary layer fluid flow on multiple solutions of and heat transfer power-law nanofluids, viscous dissipation, and permeable shrinking/stretching effects in which the shooting method was used. In this study, viscous dissipation was significant and the Brownian motion was neglected in the case of heat transfer. The results of the analysis of the mass and heat transfer in nanofluid flow in Casson fluid flow between par- allel plates with Hall current effects were obtained by Shah et al. [14] using an optimal and numerical method. The results showed that Hall currents decrease conductivity, which then increase the velocity and temperature of the fluid. Nadeem and Khan [15] obtained dual solutions of inclined stagnation point nanofluid flow with MHD over an oscillatory shrinking/stretching sheet using the fourthorder Runge-Kutta method. The results obtained indicated that dual solutions occur in both cases that is the shrinking and stretching cases. Furthermore, the obtained lower solution branch shows the same behaviour for the coefficient of skin friction in the shrinking case. On the contrary, the solution in the upper branch has perfect behaviour in both the shrinking and stretching cases. More recent studies of nanofluid flow include among others Kamal et al. [16], Besthapu et al. [17], Sadiq et al. [18], Nayak et al. [19], Yousif et al. [20], Salawu and Ogunseye [21], Kumar and Srinivas [22], Sreedevi and Reddy [23, 24], Gireesha et al. [25, 26], Archana et al. [27], and Awais et al. [28].

To the best of the authors' knowledge, the study of laminar boundary layer nanofluid flow along a fixed or moving cylinder with heat generation, radiation parameter, and slip parameter has not been widely studied. This work is aimed at studying the effects of thermal radiation, heat generation, slip effects, and other important parameters discussed in Section 4. The model of Lin and Shih [6] is modified into a laminar boundary layer nanofluid model. The fluid flow includes the effects of thermophoretic forces, thermal radiation, heat generation, magnetic field, and Brownian motion. We will focus more on the nanoparticle slip boundary condition, thermophoretic force, and Brownian motion.

The solution of the partial differential equations is obtained by the multidomain bivariate spectral quasilinearization method (MD-BSQLM). This method uses more than one domain in its collocation process; it uses the quasilinearization method based on the Newton-Raphson technique previously used by Bellman and Kalaba [29]. The obtained system of equations is used together into multiple subintervals using the spectral method based on the Chebyshev and Lagrange interpolation polynomials. These are used on the linearized nonlinear equations of PDEs independently in both directions of time and space. This approach of the MD-BSQLM gives very accurate solutions. The method yields more accurate solutions when compared to other methods such as bivariate spectral quasilinearization [30], bivariate spectral homotopy analysis method [31], and bivariate spectral relaxation method (BSRM) [32], among others.

This paper mostly focuses on the robustness and accuracy of the MD-BSQLM and in finding solutions of problems of high complexity. The robustness and accuracy of the numerical method are obtained by analysis and computations of solutions of the momentum, heat, and mass transfer systems of equations. Obtained solutions are compared to those found in the literature, and an acceptable agreement is observed. The effect of different physical parameters on the temperature, velocity, and concentration 
fields is discussed in tabular and graphical forms in Section 4. The results show the different behaviours that occur when these parameters are changed. The paper will be arranged as follows: the mathematical formulation is in Section 2, the method of solution is in Section 3, and the results will be discussed in Section 4 and conclusions in Section 5.

\section{Mathematical Formulation of the Problem}

An axisymmetric, steady boundary layer flow of an incompressible viscous fluid along with a cylinder in motion as shown in Figure 1 is considered. The cylinder is moving with a constant $U_{w}$ in a $U_{\infty}$. The $T_{w}, T_{\infty}, C_{w}$, and $C_{\infty}$ are considered. The model will consider the motion of the cylinder in both directions. The mathematical model is presented as

$$
\begin{gathered}
\frac{\partial(r u)}{\partial x}+\frac{\partial(r v)}{\partial r} \\
u \frac{\partial u}{\partial x}+v \frac{\partial u}{\partial r}=\frac{v}{r} \frac{\partial}{\partial r}\left(r \frac{\partial u}{\partial r}\right)-\frac{\sigma}{\rho} B_{0}^{2} u \\
u \frac{\partial T}{\partial x}+v \frac{\partial T}{\partial r}=\frac{k}{\rho c_{p}} \frac{1}{r} \frac{\partial}{\partial r}\left(r \frac{\partial T}{\partial r}\right)\left[1+\frac{16 T_{\infty}^{3} \sigma^{*}}{3 k^{*} k}\right]+\frac{\mu}{\rho c_{p}}\left(\frac{\partial u}{\partial r}\right)^{2} \\
+\tau\left[D_{B} \frac{\partial C}{\partial r} \frac{\partial T}{\partial r}+\frac{D_{T}}{T_{\infty}}\left(\frac{\partial T}{\partial r}\right)^{2}\right]+\frac{Q_{0}}{\rho c_{p}}\left(T-T_{\infty}\right) \\
u \frac{\partial C}{\partial x}+v \frac{\partial C}{\partial r}=D_{B} \frac{1}{r} \frac{\partial}{\partial r}\left(r \frac{\partial C}{\partial r}\right)+\frac{D_{T}}{T_{\infty}} \frac{1}{r} \frac{\partial}{\partial r}\left(r \frac{\partial T}{\partial r}\right)
\end{gathered}
$$

subject to the boundary conditions

$$
\begin{gathered}
u=U_{w}(x)+B_{1} v \frac{\partial u}{\partial r}, v=0, T=T_{w}(x), C=C_{w}(x), \text { at } r=R \\
u \longrightarrow U_{\infty}, T \longrightarrow T_{\infty}, C \longrightarrow C_{\infty} \text { as } r \longrightarrow \infty
\end{gathered}
$$

To solve this problem, we introduce the following nondimensional groups and variables:

$$
\begin{gathered}
\xi(x)=\frac{4}{R}\left(\frac{v x}{U}\right)^{1 / 2}, \\
\eta(x, r)=\frac{r^{2}-R^{2}}{\xi R^{2}}, \\
\psi(x, r)=R(v x u)^{1 / 2} f(\xi, \eta),
\end{gathered}
$$

$$
\begin{gathered}
\theta(\eta, \xi)=\frac{T-T_{\infty}}{T_{w}-T_{\infty}}, \\
\phi(\eta, \xi)=\frac{C-C_{\infty}}{C_{w}-C_{\infty}},
\end{gathered}
$$

where $\psi$ is the stream function which is defined as

$$
\begin{aligned}
& u=\frac{1}{r} \frac{\partial \psi}{\partial r} \\
& v=\frac{1}{r} \frac{\partial \psi}{\partial x},
\end{aligned}
$$

which satisfies the continuity equation (1). Substituting equation (9) in equations (2)-(5) gives

$$
\begin{aligned}
& (1+\xi \eta) f^{\prime \prime \prime}+\xi f^{\prime \prime}-4 M f^{\prime}+\xi\left[f^{\prime \prime} \frac{\partial f}{\partial \xi}-f^{\prime} \frac{p f^{\prime}}{\partial \xi}\right]=0, \\
& \frac{1}{\operatorname{Pr}}\left(1+N_{r}\right)\left[(1+\xi \eta) \theta^{\prime \prime}+\xi \theta^{\prime}\right]+f \theta^{\prime}-2 m \theta f^{\prime} \\
& +(1+\xi \eta)\left[\mathrm{Ec}\left(f^{\prime \prime}\right)^{2}+N_{b} \theta^{\prime} \phi^{\prime}+N_{t}\left(\theta^{\prime}\right)^{2}\right]+\mathrm{He} \theta \\
& +\xi\left[\theta^{\prime} \frac{\partial f}{\partial \xi}-f^{\prime} \frac{\partial \theta}{\partial \xi}\right]=0 \\
& \frac{1}{\mathrm{Sc}}\left[(1+\xi \eta) \phi^{\prime \prime}+\xi \phi^{\prime}\right]+f \phi^{\prime}-2 r \phi f^{\prime} \\
& +\frac{N_{t}}{N_{b}} \frac{1}{\mathrm{Sc}}\left[(1+\xi \eta) \theta^{\prime \prime}+\xi \theta^{\prime}\right]+\xi\left[\theta^{\prime} \frac{\partial f}{\partial \xi}-f^{\prime} \frac{\partial \theta}{\partial \xi}\right]=0
\end{aligned}
$$

subject to the boundary conditions:

$$
\begin{gathered}
f^{\prime}(0)=2+\delta f^{\prime \prime}(0), f(0)=0, f^{\prime}(\infty)=0 \\
\theta(0)=1, \theta(\infty)=0 \\
\phi(0)=1, \phi(\infty)=0
\end{gathered}
$$

where prime defines derivatives with respect to $\eta$, magnetic parameter is $M=\left(\sigma B_{0}^{2} x\right) /(\rho u)$, Prandtl number is $\operatorname{Pr}=\left(\rho c_{p}\right) /(k)$, thermal radiation parameter is $N_{r}=\left(16 T_{\infty}^{3}\right.$ $\left.\sigma^{*}\right) /\left(3 k^{*} k\right)$, Eckert number is $\mathrm{Ec}=\left(U^{2}\right) /\left(4 c_{p} A x^{m}\right), \mathrm{Ec}=($ $\left.U^{2}\right) /\left(4 c_{p}\left(T_{w}-T_{\infty}\right)\right)$, nondimensional heat generation parameter is $\mathrm{He}=\left(4 Q_{0} x\right) /\left(\rho c_{p} U\right)$, Brownian motion parameter is $N_{b}=\left(\tau D_{B}\left(C_{w}-C_{\infty}\right)\right) /(v)$, thermophoresis parameter is $N_{t}=\left(\tau D_{T}\left(T_{w}-T_{\infty}\right)\right) /\left(\nu T_{\infty}\right)$, Schmidt number is $S c=(v) /\left(D_{B}\right)$, and nondimensional velocity slip parameter is $\delta=\left(\beta_{1} / 2\right)(\nu u / x)^{1 / 2}$. 


\section{Multidomain Bivariate Spectral Quasilinearization Method}

In this section, we describe how to apply the multidomain bivariate spectral quasilinearization method (MD-BSQLM) for solving the system of coupled PDEs that are highly coupled as shown in equations (13)-(15). The solution is decomposed into a large interval with small subdivisions. The solution is evaluated at each time step at the end of each subinterval. The multidomain approach is applied in the $\xi$ direction. The system of equations is first linearized using the quasilinearization (QLM) as indicated in [29, 33]. The QLM approach makes use of the Taylor series approximation to linearize the system of equations. In this method, we assume that the value of the function on the current and previous iterations is small. Applying the QLM on (13)-(15) gives the following:

$$
\begin{gathered}
a_{0, s} f_{s+1}{ }^{\prime \prime \prime}+a_{1, s} f_{s+1}{ }^{\prime \prime}+a_{2, s} f_{s+1}{ }^{\prime}+a_{3, s} f_{s+1}+a_{4, s} \frac{\partial f_{s+1}^{\prime}}{\partial \xi}++a_{5, s} \frac{\partial f_{s+1}}{\partial \xi}=R_{1, s}, \\
b_{0, s} \theta_{s+1}{ }^{\prime \prime}+b_{1, s} \theta_{s+1}{ }^{\prime}+b_{2, s} \theta_{s+1}+b_{3, s} \frac{\partial \theta_{s+1}}{\partial \xi}+b_{4, s} f^{\prime \prime}{ }_{s+1} \\
+b_{5, s} f^{\prime}{ }_{s+1}+b_{6, s} f_{s+1}+b_{7, s} \frac{\partial f_{s+1}}{\partial \xi}+b_{8, s} \phi_{s+1}^{\prime}=R_{2, s}, \\
c_{0, s} \phi_{s+1}{ }^{\prime \prime}+c_{1, s} \phi_{s+1}{ }^{\prime}+c_{2, s} \phi_{s+1}+c_{3, s} \frac{\partial \phi_{s+1}}{\partial \xi}+c_{4, s} f^{\prime}{ }_{s+1}+c_{5, s} f_{s+1} \\
+c_{6, s} \frac{\partial f_{s+1}}{\partial \xi}+c_{7, s} \theta_{s+1}^{\prime \prime}+c_{8, s} \theta_{s+1}^{\prime}=R_{3, s},
\end{gathered}
$$

where

$$
\begin{gathered}
a_{0, s}=1+\xi \eta, \\
a_{1, s}=\xi+f_{s}+\xi \frac{\partial f_{s}}{\partial \xi}, \\
a_{2, s}=-M-\xi \frac{\partial f_{s}^{\prime}}{\partial \xi}, \\
a_{3, s}=-f^{\prime \prime}{ }_{s}^{\prime} \\
a_{4, s}=-\xi f_{s}^{\prime} \\
b_{0, s}=\frac{1}{\operatorname{Pr}}(1+\xi \eta), \\
b_{1, s}=\frac{1}{\operatorname{Pr}} \xi+f_{s}+(1+\xi \eta) N_{b} \phi_{s}^{\prime}+2 N_{t}(1+\xi \eta) \phi_{r}^{\prime}+\xi \frac{\partial f_{s}}{\partial \xi}, \\
b_{2, r}=-2 m f_{s}^{\prime}+\mathrm{He}, \\
b_{3, s}=-\xi f_{s}^{\prime}, \\
b_{4, s}=2 \mathrm{Ec}(1+\xi \eta) f_{r}^{\prime \prime},
\end{gathered}
$$

$$
\begin{aligned}
& b_{5, s}=-2 m \theta_{s}-\xi \frac{\partial \theta}{\partial \xi}, \\
& b_{6, s}=\theta_{s}^{\prime}, \\
& b_{7, s}=\xi \theta_{s}^{\prime}, \\
& b_{8, s}=(1+\xi \eta) N_{b} \theta_{s}^{\prime}, \\
& c_{0, s}=\frac{1}{\mathrm{Sc}}(1+\xi \eta), \\
& c_{1, s}=\frac{1}{\mathrm{Sc}} \xi+f_{s}+\xi \frac{\partial f_{s}}{\partial \xi}, \\
& c_{2, s}=-2 r f_{s}^{\prime} \text {, } \\
& c_{3, s}=-\xi f^{\prime}, \\
& c_{4, s}=-2 r \phi_{s}-\xi \frac{\partial \phi_{s}}{\partial \xi}, \\
& c_{5, s}=\phi_{s}^{\prime}, \\
& c_{6, s}=\xi \phi_{s}^{\prime}, \\
& c_{7, s}=\frac{N_{t}}{N_{b}} \frac{1}{\mathrm{Sc}}(1+\xi \eta), \\
& c_{8, s}=\frac{N_{t}}{N_{b}} \frac{1}{\mathrm{Sc}} \xi, \\
& R_{1, s}=f^{\prime \prime} f_{s}+\frac{\partial f_{s}}{\partial \xi} f^{\prime \prime}{ }_{s}-\frac{\partial f^{\prime}}{\partial \xi},
\end{aligned}
$$

$$
\begin{gathered}
R_{2, s}=\theta_{s}^{\prime} f_{s}-2 m f_{s}^{\prime} \theta_{s}+(1+\xi \eta) \operatorname{Ec}\left(f^{\prime \prime}{ }_{s}\right)^{2}+(1+\xi \eta) N_{b} \phi_{s}^{\prime} \theta_{s}^{\prime} \\
+(1+\xi \eta) N_{t}\left(\theta^{\prime}{ }_{r}\right)^{2}+\xi \frac{\partial f_{s}}{\partial \xi} \theta_{r}^{\prime}{ }_{r}-\xi \frac{\partial \theta_{f}}{\partial \xi} f_{s}^{\prime} \\
R_{3, s}=\phi^{\prime}{ }_{s} f_{s}-2 r f_{s}^{\prime} \phi_{s}+\xi \frac{\partial f_{s}}{\partial \xi} \phi_{s}^{\prime}-\xi \frac{\partial \phi_{s}}{\partial \xi} f_{s}^{\prime} .
\end{gathered}
$$

Now, let $\xi \in \Omega$, where $\Omega \in[0, T]$ and the domain $\Omega$ is decomposed into $p$ nonoverlapping intervals as

$$
\Omega_{m}=\left[\xi_{m-1}, \xi_{m}\right], \xi_{m-1}<\xi_{m}, \xi_{0}=0, \xi_{p}=T, m=1,2, \cdots, p .
$$

The PDEs are solved separately at each of the subintervals $p$. Solutions of the $m$ th interval are obtained by using solutions from the $m-1$ th interval. This process is implemented as follows:

$$
\begin{aligned}
& a_{0, s}^{(m)} f_{s+1}^{\prime \prime \prime}+a_{1, s}^{(m)} f_{s+1}{ }^{\prime \prime(m)}+a_{2, s}^{(m)} f_{r+1}{ }^{\prime(m)}+a_{3, s}^{(m)} f_{s+1}^{(m)} \\
& +a_{4, s}^{(m)} \frac{\partial f_{s+1}^{\prime(m)}}{\partial \xi}+a_{5, s}^{(m)} \frac{\partial f_{s+1}^{(m)}}{\partial \xi}=R_{1, s}^{(m)},
\end{aligned}
$$




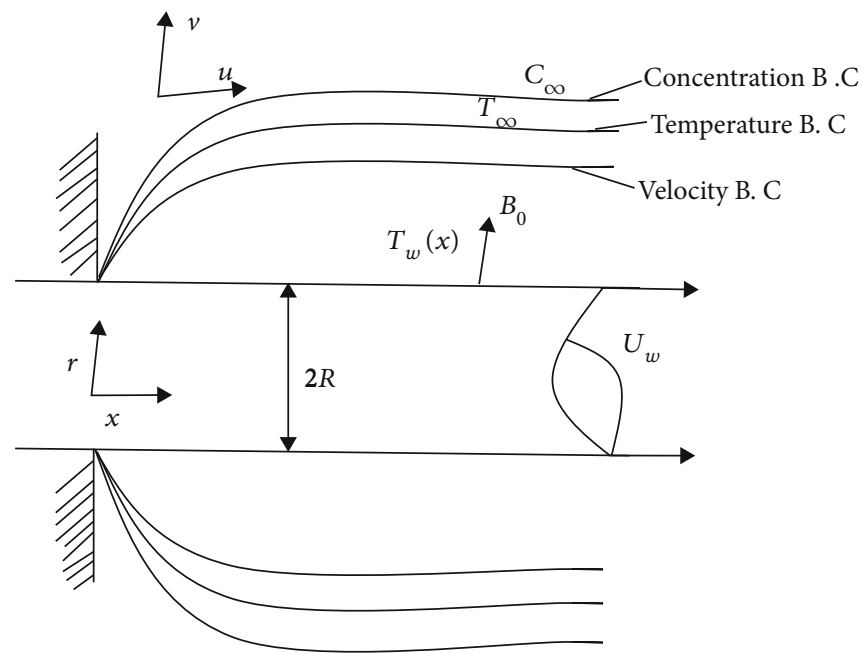

Figure 1: The flow coordinate system and the flow configuration.

TABLE 1: Comparison of the MD-BSQLM approximate solutions of $f^{\prime \prime}(0, \xi)$, against those of Ref. [36] for different values of $\lambda$ and $\xi$ when $M=0, \operatorname{Pr}=0.72$, and $N_{r}=\delta=\mathrm{He}=m=r=0$ and in the absence of $N_{t}, N_{b}$, and Sc.

\begin{tabular}{lcccc}
\hline$\xi$ & \multicolumn{2}{c}{$f^{\prime \prime}(0, \xi)[36]$} & \multicolumn{2}{c}{$f^{\prime \prime}(0, \xi)$ (present) } \\
& $\lambda=0$ & $\lambda=1$ & $\lambda=0$ & $\lambda=1$ \\
\hline 0 & 1.328229 & -1.774973 & 1.328230 & -1.774993 \\
0.0001 & 1.328286 & -1.775009 & 1.328287 & -1.775024 \\
0.0005 & 1.328515 & -1.775139 & 1.328515 & -1.775149 \\
0.001 & 1.328801 & -1.775292 & 1.328801 & -1.775305 \\
0.005 & 1.331084 & -1.776544 & 1.331084 & -1.776554 \\
0.01 & 1.333934 & -1.778103 & 1.333935 & -1.778115 \\
0.05 & 1.356475 & -1.790768 & 1.356575 & -1.790609 \\
0.1 & 1.384492 & -1.806219 & 1.384491 & -1.806235 \\
0.5 & 1.594957 & -1.931348 & 1.595055 & -1.931760 \\
1 & 1.834846 & -2.103250 & 1.834850 & -2.090697 \\
1.5 & 2.056828 & -2.243225 & 2.056913 & -2.250873 \\
2 & 2.266093 & -2.412581 & 2.266166 & -2.411156 \\
\hline
\end{tabular}

$$
\begin{aligned}
& b_{0, s}^{(m)} \theta_{s+1}{ }^{\prime(m)}+b_{1, s}^{(m)} \theta_{s+1}{ }^{\prime(m)}+b_{2, s}^{(m)} \theta_{s+1}^{(m)}+b_{3, r}^{(m)} \frac{\partial \theta_{s+1}^{(m)}}{\partial \xi} \\
& +b_{4, s}^{(m)} f_{s+1}{ }^{\prime(m)}+b_{5, s}^{(m)} f_{s+1}{ }^{\prime(m)}+b_{6, s}^{(m)} f_{s+1}^{(m)}+b_{7, s}^{(m)} \frac{\partial f_{s+1}^{(m)}}{\partial \xi} \\
& +b_{8, s}^{(m)} \phi_{s+1}{ }^{\prime(m)}=R_{2, s}^{(m)},
\end{aligned}
$$

$$
\begin{aligned}
& c_{0, s}^{(m)} \phi_{s+1}{ }^{\prime \prime(m)}+c_{1, s}^{(m)} \phi_{s+1}{ }^{\prime(m)}+c_{2, s}^{(m)} \phi_{s+1}^{(m)}+c_{3, s}^{(m)} \frac{\partial \phi_{s+1}^{(m)}}{\partial \xi} \\
& +c_{4, s}^{(m)} f_{s+1}{ }^{\prime(m)}+c_{5, r}^{(m)} f_{r+1}^{(m)}+c_{6, s}^{(m)} \frac{\partial f_{s+1}^{(m)}}{\partial \xi}+c_{7, s}^{(m)} \theta_{s+1}{ }^{\prime(m)} \\
& +c_{7, s}^{(m)} \theta_{s+1}{ }^{\prime(m)}=R_{3, s}^{(m)},
\end{aligned}
$$

the boundary conditions are imposed

$$
\begin{gathered}
f_{s+1}{ }^{\prime(m)}(0, \xi)=2+\delta f_{s+1}{ }^{\prime(m)}(0, \xi) \\
{f_{s+1}{ }^{\prime(m)}}^{(m}(0, \xi)=0 \\
f_{s+1}{ }^{\prime(m)}(\infty, \xi)=0 \\
\theta_{s+1}^{\prime(m)}(0, \xi)=1 \\
\theta_{s+1}^{(m)}(\infty, \xi)=0 \\
\phi_{s+1}^{\prime(m)}(0, \xi)=1, \phi_{s+1}^{(m)}(\infty, \xi)=0 .
\end{gathered}
$$

An appropriate initial guess is imposed to start the multidomain iteration. This gives the solution in the first interval and satisfies the boundary condition (16). The initial conditions at the next interval are prescribed by the conditions that ensure continuity as follows:

$$
\begin{aligned}
& f^{(m)}\left(\eta, \xi_{m-1}\right)=f^{(m-1)}\left(\eta, \xi_{m-1}\right), \\
& \theta^{(m)}\left(\eta, \xi_{m-1}\right)=\theta^{(m-1)}\left(\eta, \xi_{m-1}\right), \\
& \phi^{(m)}\left(\eta, \xi_{m-1}\right)=\phi^{(m-1)}\left(\eta, \xi_{m-1}\right) .
\end{aligned}
$$

The domains in the $\eta$ and $\xi$ directions have transformed the domain $(x, t) \in[-1,1] \times[-1,1]$ for computation, at each subinterval using the linear transformation

$$
\begin{gathered}
\eta=\frac{L x}{2}(1+x), \\
\xi=\frac{1}{2}\left(\xi_{m}-\xi_{m-1}\right) t+\frac{1}{2}\left(\xi_{m}+\xi_{m-1}\right),
\end{gathered}
$$

where $L x$ is a number that approximates infinity in $\eta$. The Chebyshev-Gauss-Lobatto nodes defined in $[34,35]$ 


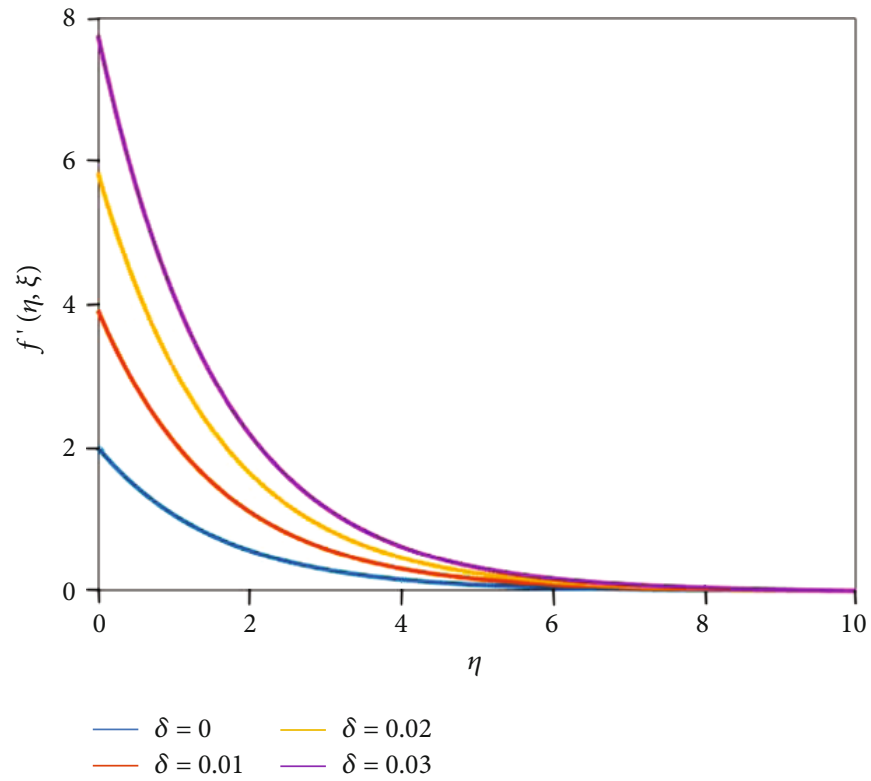

Figure 2: Velocity profile for different values of $\delta$ when $\lambda=0.1, N=0.5, n=0.5, f_{w}=0.8, \xi=1, N_{b}=N_{t}=D f=B i=S r=0.5$, He $=\delta=N R$ $=0.2, \mathrm{Sc}=0.6$, and $\operatorname{Pr}=1$.

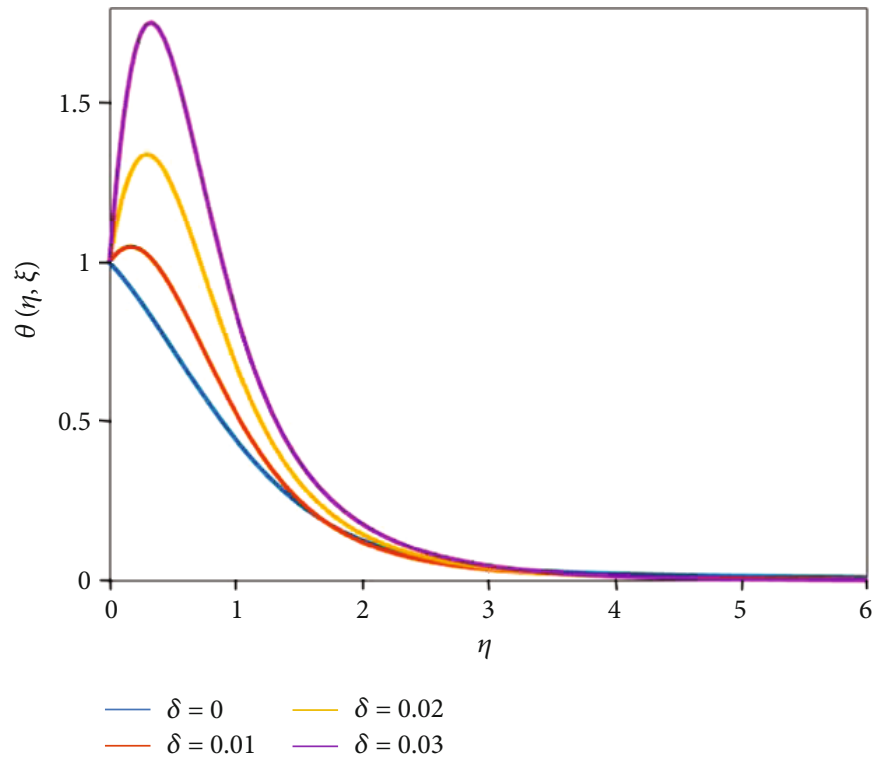

Figure 3: Temperature profile for different values of $\lambda$ when $D e=0.1, N=0.5, n=0.5, f_{w}=0.8, \xi=1, N_{b}=N_{t}=D f=S r=B i=0.5$, He $=\delta$ $=N R=0.2, \mathrm{Sc}=0.6$, and $\operatorname{Pr}=1$.

are used for collocation as follows:

$x_{i}=\cos \left(\frac{\pi i}{N_{x}}\right), t_{j}=\cos \left(\frac{\pi j}{N_{t}}\right), i=0,1, \cdots, N_{x}, j=0,1, \cdots, N_{t}, x \in[-1,1], t \in[-1,1]$,

where $\left(N_{x}+1\right)$ and $\left(N_{t}+1\right)$ are all the points in $\eta$ - and $\xi$ -directions used for collocation, respectively.

If the solutions $f, \theta$, and $\phi$ are approximated at each subinterval by using the bivariate Lagrange interpolation poly- nomial of the form

$$
f^{(m)}(\eta, \xi) \approx F^{(m)}(x, t)=\sum_{p=0}^{N_{x}} \sum_{q=0}^{N_{t}} F^{(m)}\left(x_{p}, t_{q}\right) L_{p}(x) L_{q}(t)
$$

$$
\theta^{(m)}(\eta, \xi) \approx \Theta^{(m)}(x, t)=\sum_{p=0}^{N_{x}} \sum_{q=0}^{N_{t}} \Theta^{(m)}\left(x_{p}, t_{q}\right) L_{p}(x) L_{q}(t)
$$




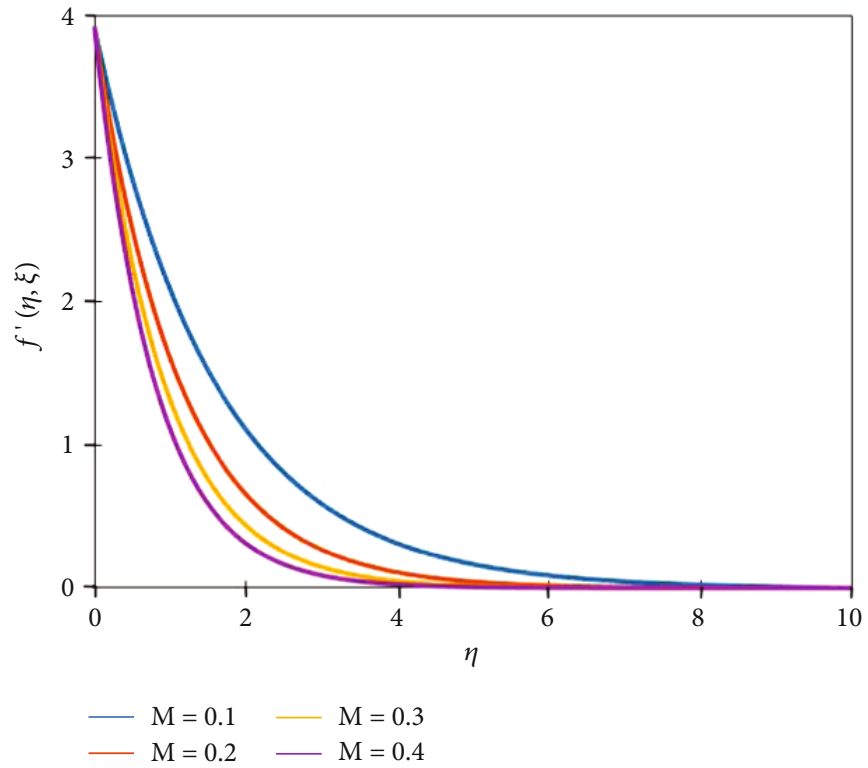

Figure 4: Velocity profile for different values of $M$ when $\lambda=0.1, N=0.5, n=0.5, f_{w}=0.8, \xi=1, N_{b}=N_{t}=D f=B i=S r=0.5, \mathrm{He}=\delta=N$ $R=0.2, \mathrm{Sc}=0.6$, and $\mathrm{Pr}=1$.

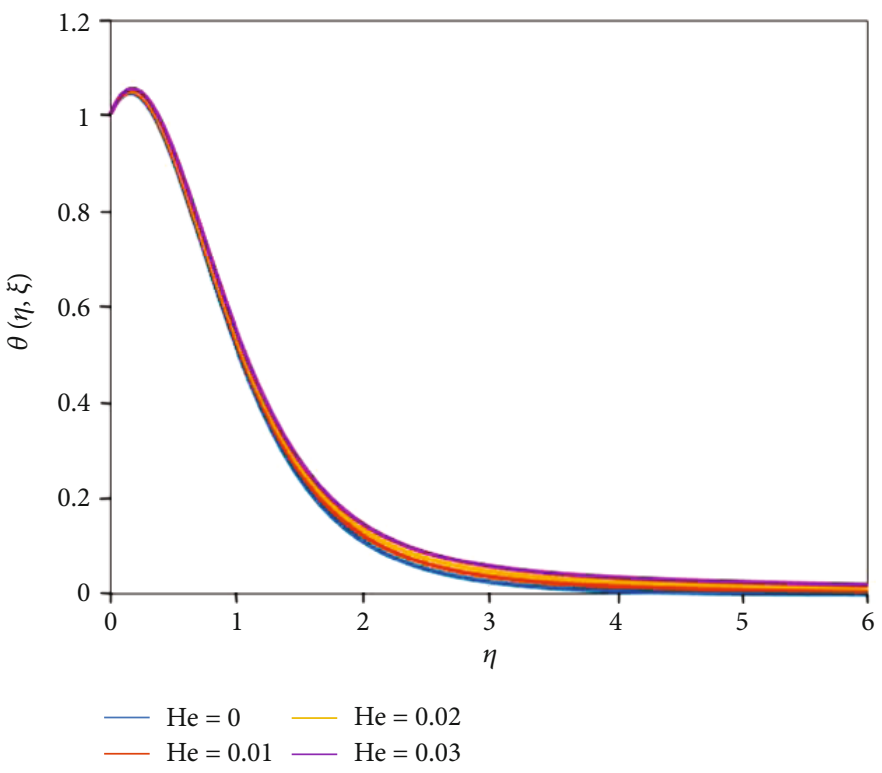

Figure 5: Temperature profile for different values of He when $D e=0.1, N=0.5, n=0.5, f_{w}=0.8, \xi=1, N_{b}=N_{t}=\mathrm{D} f=S r=B i=0.5, \mathrm{He}=$ $\delta=N R=0.2, \mathrm{Sc}=0.6$, and $\operatorname{Pr}=1$.

$$
\phi^{(m)}(\eta, \xi) \approx \Phi^{(m)}(x, t)=\sum_{p=0}^{N_{x}} \sum_{q=0}^{N_{t}} \Phi^{(m)}\left(x_{p}, t_{q}\right) L_{p}(x) L_{q}(t)
$$

where the Lagrange cardinal polynomial functions $L_{p}(x)$ and $L_{q}(t)$ are defined as

$$
L_{p}(x)=\prod_{\substack{i=0 \\ i \neq k}}^{N_{x}} \frac{x-x_{k}}{x_{i}-x_{k}}
$$

$$
L_{q}(t)=\prod_{\substack{j=0 \\ j \neq k}}^{N_{t}} \frac{t-t_{k}}{t_{j}-t_{k}},
$$




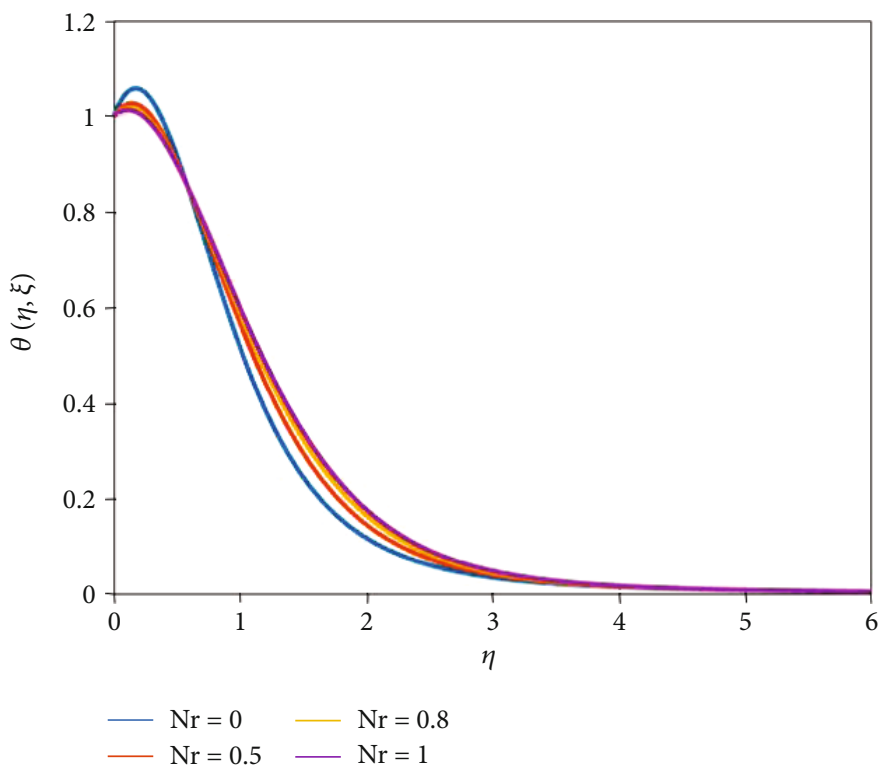

Figure 6: Temperature profile for different values of $N_{r}$ when $\lambda=0.1, N=0.5, n=0.5, f_{w}=0.8, \xi=1, N_{b}=N_{t}=D f=B i=S r=0.5, \mathrm{He}=\delta$ $=N R=0.2, \mathrm{Sc}=0.6$, and $\operatorname{Pr}=1$.

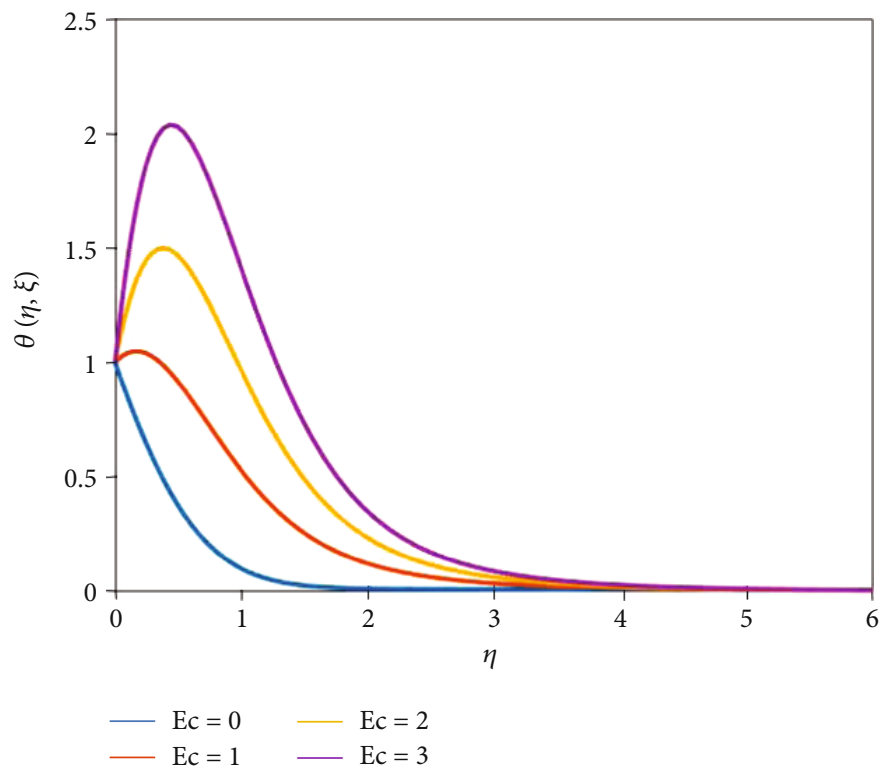

Figure 7: Temperature profile for different values of Ec when $\lambda=0.1, N=0.5, n=0.5, f_{w}=0.8, \xi=1, N_{b}=N_{t}=D f=B i=S r=0.5, \mathrm{He}=\delta$ $=N R=0.2, \mathrm{Sc}=0.6$, and $\operatorname{Pr}=1$.

with

$$
\begin{aligned}
& L_{p}\left(x_{k}\right)=\delta_{i k}= \begin{cases}0 & \text { if } i \neq k, \\
1 & \text { if } i=k,\end{cases} \\
& L_{q}\left(t_{k}\right)=\delta_{j k}= \begin{cases}0 & \text { if } j \neq k, \\
1 & \text { if } j=k\end{cases}
\end{aligned}
$$

$, 1,2, \cdots, N_{x}$, the derivative of $f, \theta$, and $\phi$ with respect to $\eta$ is evaluated as

$$
\begin{aligned}
\frac{\partial f^{(m)}}{\partial \eta}\left(x_{i}, t_{j}\right) & =\sum_{p=0}^{N_{x}} \sum_{q=0}^{N_{t}} F^{(m)}\left(x_{p}, t_{q}\right) \frac{d L_{p}\left(x_{i}\right)}{d x} L_{p}\left(t_{j}\right)=\sum_{p=0}^{N_{x}} F^{(m)}\left(x_{p}, t_{j}\right) \frac{d L_{p}\left(x_{i}\right)}{d x} \\
& =\sum_{p=0}^{N_{x}}\left(\frac{2}{L_{x}}\right) \hat{D}_{i, p} F^{(m)}\left(x_{p}, t_{j}\right)=\left(\frac{2}{L_{x}}\right) \hat{\mathbf{D}} \mathbf{F}_{j}^{(m)}=\mathbf{D F}_{j}^{(m)},
\end{aligned}
$$

At the Chebyshev-Gauss-Lobatto points $\left(x_{i}, t_{j}\right)$ for $i=0$ 


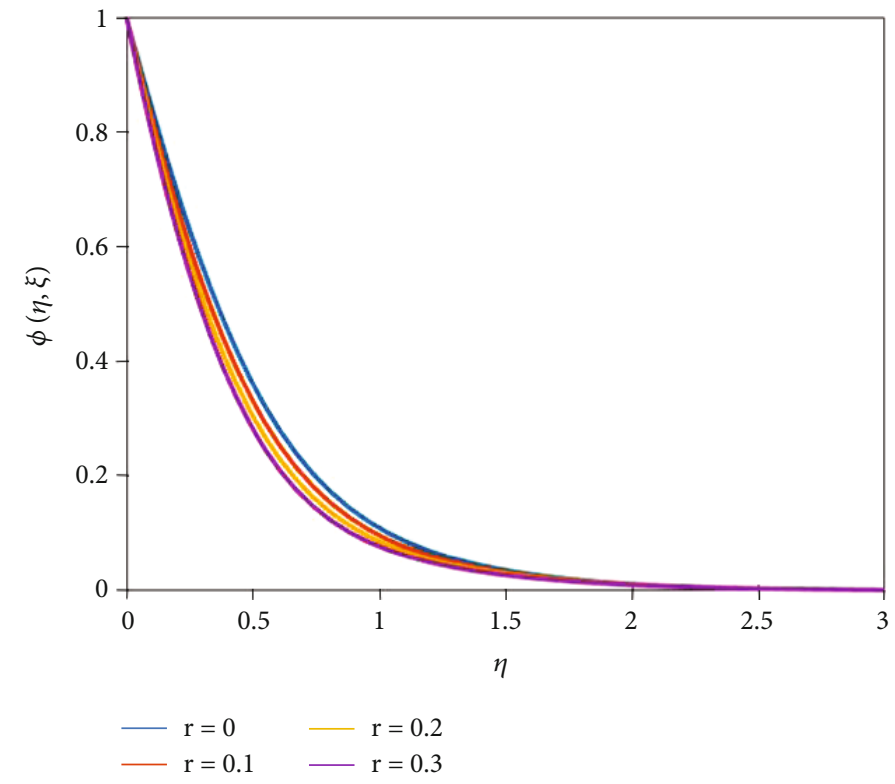

Figure 8: Concentration profile for different values of $r$ when $D e=0.1, N=0.5, n=0.5, f_{w}=0.8, \xi=1, N_{b}=N_{t}=D f=S r=B i=0.5, \mathrm{He}=$ $\delta=N R=0.2, \mathrm{Sc}=0.6$, and $\operatorname{Pr}=1$.

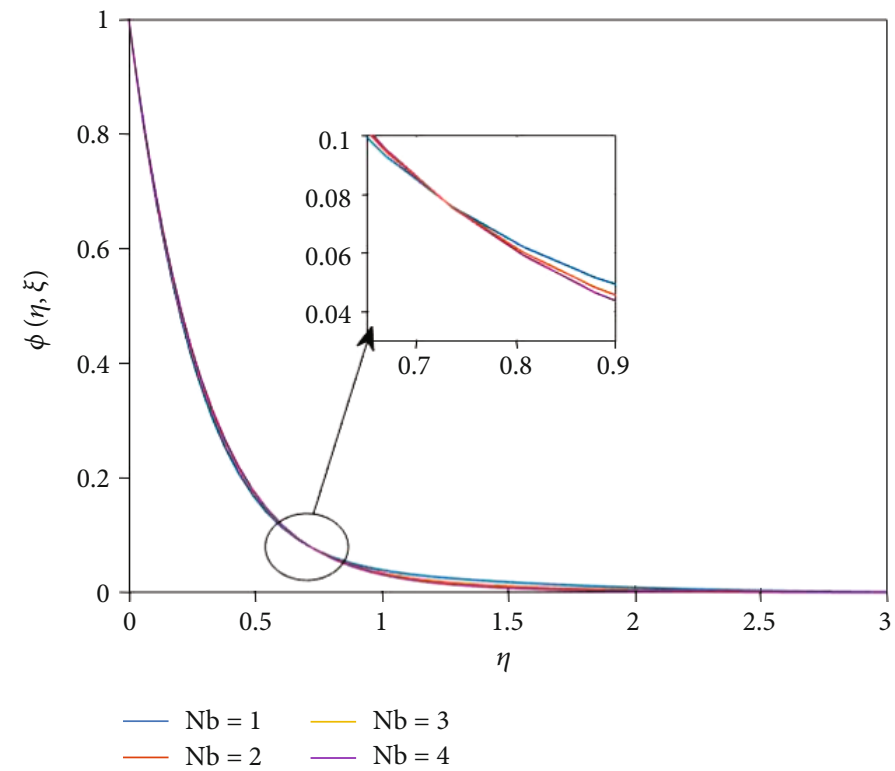

Figure 9: Concentration profile for different values of $N_{b}$ when $\lambda=0.1, N=0.5, n=0.5, f_{w}=0.8, \xi=1, N_{b}=N_{t}=D f=B i=S r=0.5, \mathrm{He}=$ $\delta=N R=0.2, \mathrm{Sc}=0.6$, and $\mathrm{Pr}=1$.

$$
\begin{aligned}
\frac{\partial \theta^{(m)}}{\partial \eta}\left(x_{i}, t_{j}\right) & =\sum_{p=0}^{N_{x}} \sum_{q=0}^{N_{t}} \Theta^{(m)}\left(x_{p}, t_{q}\right) \frac{d L_{p}\left(x_{i}\right)}{d x} L_{p}\left(t_{j}\right) \\
& =\sum_{p=0}^{N_{x}} \Theta^{(m)}\left(x_{p}, t_{j}\right) \frac{d L_{p}\left(x_{i}\right)}{d x}=\sum_{p=0}^{N_{x}}\left(\frac{2}{L_{x}}\right) \widehat{D}_{i, p} \Theta^{(m)}\left(x_{p}, t_{j}\right) \\
& =\left(\frac{2}{L_{x}}\right) \widehat{\mathbf{D}} \Theta_{j}^{(m)}=\mathbf{D} \Theta_{j}^{(m)},
\end{aligned}
$$$$
\frac{\partial \phi^{(m)}}{\partial \eta}\left(x_{i}, t_{j}\right)=\sum_{p=0}^{N_{x}} \sum_{q=0}^{N_{t}} \Phi^{(m)}\left(x_{p}, t_{q}\right) \frac{d L_{p}\left(x_{i}\right)}{d x} L_{p}\left(t_{j}\right)
$$$$
=\sum_{p=0}^{N_{x}} \Phi^{(m)}\left(x_{p}, t_{j}\right) \frac{d L_{p}\left(x_{i}\right)}{d x}=\sum_{p=0}^{N_{x}}\left(\frac{2}{L_{x}}\right) \widehat{D}_{i, p} \Phi^{(m)}\left(x_{p}, t_{j}\right)
$$$$
=\left(\frac{2}{L_{x}}\right) \hat{\mathbf{D}} \Phi_{j}^{(m)}=\mathbf{D} \Phi_{j}^{(m)},
$$ 


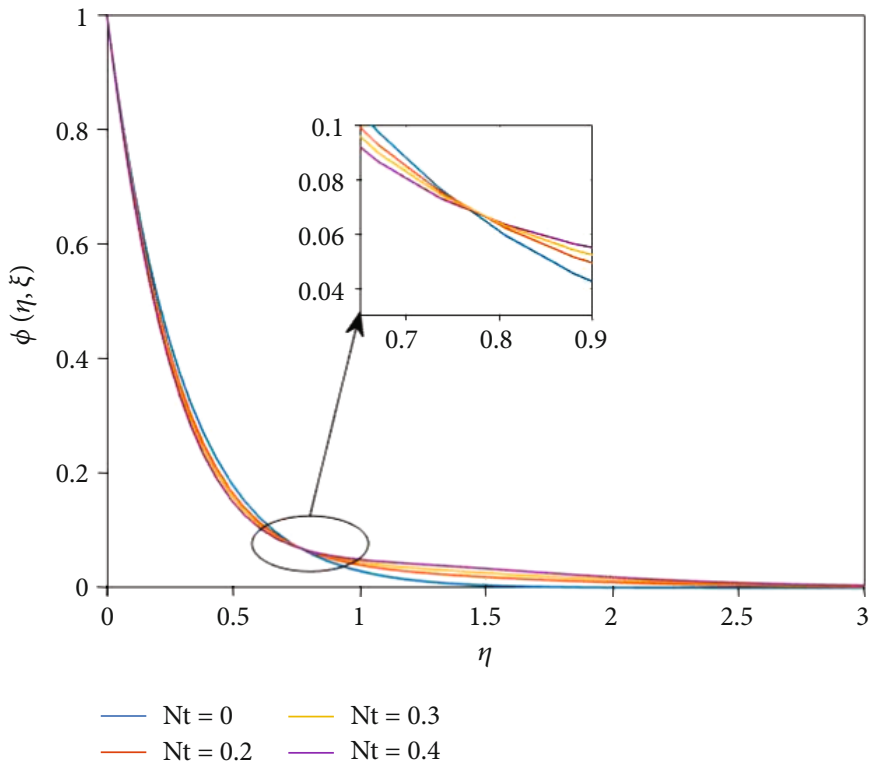

FIGURE 10: Concentration profile for different values of $N_{t}$ when $D e=0.1, N=0.5, n=0.5, f_{w}=0.8, \xi=1, N_{b}=N_{t}=D f=S r=B i=0.5, \mathrm{He}$ $=\delta=N R=0.2, \mathrm{Sc}=0.6$, and $\operatorname{Pr}=1$.

where $\widehat{\mathbf{D}}=L_{x} \mathbf{D} / 2$ is the differentiation matrix of size ( $\left.N_{x}+1\right) \times\left(N_{x}+1\right)$ as indicated in Trefethen [34]. The matrices $\mathbf{F}_{j}^{(m)}, \boldsymbol{\Theta}_{j}^{(m)}$, and $\boldsymbol{\Phi}_{j}^{(m)}$ are defined as

$$
\begin{aligned}
\mathbf{F}_{j}^{(m)} & =\left[F\left(\eta_{0}, \xi_{j}\right), F\left(\eta_{1}, \xi_{j}\right) \cdot F\left(\eta_{N_{x}}, \xi_{j}\right)\right]^{T}, \\
\boldsymbol{\Theta}_{j}^{(m)} & =\left[\Theta\left(\eta_{0}, \xi_{j}\right), \Theta\left(\eta_{1}, \xi_{j}\right) \cdot \Theta\left(\eta_{N_{x}}, \xi_{j}\right)\right]^{T}, \\
\boldsymbol{\Phi}_{j}^{(m)} & =\left[\Phi\left(\eta_{0}, \xi_{j}\right), \Phi\left(\eta_{1}, \xi_{j}\right) \cdot \Phi\left(\eta_{N_{x}}, \xi_{j}\right)\right]^{T},
\end{aligned}
$$

where $T$ denotes transpose of the matrix.

The derivative of order $n$ for $f, \theta$, and $\phi$ w.r.t $\eta$ are approximated as follows:

$$
\begin{gathered}
\frac{\partial^{n} F^{(m)}}{\partial \eta^{n}}\left(x_{i}, t_{j}\right)=\mathbf{D}^{(n)} \mathbf{F}_{j}^{(m)}, \\
\frac{\partial^{n} F^{(m)}}{\partial \eta^{n}}\left(x_{i}, t_{j}\right)=\mathbf{D}^{(n)} \boldsymbol{\Theta}_{j}^{(m)}, \\
\frac{\partial^{n} \Phi^{(m)}}{\partial \eta^{n}}\left(x_{i}, t_{j}\right)=\mathbf{D}^{(n)} \boldsymbol{\Phi}_{j}^{(m)} .
\end{gathered}
$$

The derivatives of $f, \theta$, and $\phi$ are evaluated at each point $\left(x_{i}, t_{j}\right)$ for $j=0,1,2, \cdots, N_{t}$ as

$$
\begin{aligned}
\frac{\partial F^{(m)}}{\partial \xi}\left(x_{i}, t_{j}\right) & =\sum_{p=0}^{N_{x}} \sum_{q=0}^{N_{t}} F^{(m)}\left(x_{p}, t_{q}\right) L_{p}\left(x_{i}\right) \frac{d L_{q}\left(t_{j}\right)}{d t} \\
& =\sum_{q=0}^{N_{t}} F^{(m)}\left(x_{i}, t_{q}\right) \frac{d L_{q}\left(t_{j}\right)}{d t},
\end{aligned}
$$

$$
\begin{aligned}
& \sum_{q=0}^{N_{t}}\left(\frac{2}{\xi_{m}-\xi_{m-1}}\right) \hat{d}_{j, q} F^{(m)}\left(x_{i}, t_{q}\right)=\sum_{q=0}^{N_{t}}\left(\frac{2}{\xi_{m}-\xi_{m-1}}\right) \hat{d}_{j, q} F_{q}^{(m)} \\
&=\sum_{q=0}^{N_{t}} d_{j, q} F_{q}^{(m)} \\
& \frac{\partial \Theta^{(m)}}{\partial \xi}\left(x_{i}, t_{j}\right)=\sum_{p=0}^{N_{x}} \sum_{q=0}^{N_{t}} \Theta^{(m)}\left(x_{p}, t_{q}\right) L_{p}\left(x_{i}\right) \frac{d L_{q}\left(t_{j}\right)}{d t} \\
&=\sum_{q=0}^{N_{t}} \Theta^{(m)}\left(x_{i}, t_{q}\right) \frac{d L_{q}\left(t_{j}\right)}{d t},
\end{aligned}
$$

$$
\begin{aligned}
& \sum_{q=0}^{N_{t}}\left(\frac{2}{\xi_{m}-\xi_{m-1}}\right) \hat{d}_{j, q} \Theta^{(m)}\left(x_{i}, t_{q}\right)=\sum_{q=0}^{N_{t}}\left(\frac{2}{\xi_{m}-\xi_{m-1}}\right) \hat{d}_{j, q} \Theta_{q}^{(m)} \\
& \quad=\sum_{q=0}^{N_{t}} d_{j, q} \Theta_{q}^{(m)},
\end{aligned}
$$

$$
\begin{aligned}
\frac{\partial \Phi^{(m)}}{\partial \xi}\left(x_{i}, t_{j}\right) & =\sum_{p=0}^{N_{x}} \sum_{q=0}^{N_{t}} \Phi^{(m)}\left(x_{p}, t_{q}\right) L_{p}\left(x_{i}\right) \frac{d L_{q}\left(t_{j}\right)}{d t} \\
& =\sum_{q=0}^{N_{t}} \Phi^{(m)}\left(x_{i}, t_{q}\right) \frac{d L_{q}\left(t_{j}\right)}{d t}
\end{aligned}
$$

$$
\begin{aligned}
& \sum_{q=0}^{N_{t}}\left(\frac{2}{\xi_{m}-\xi_{m-1}}\right) \hat{d}_{j, q} \Phi^{(m)}\left(x_{i}, t_{q}\right)=\sum_{q=0}^{N_{t}}\left(\frac{2}{\xi_{m}-\xi_{m-1}}\right) \hat{d}_{j, q} \Phi_{q}^{(m)} \\
& \quad=\sum_{q=0}^{N_{t}} d_{j, q} \Phi_{q}^{(m)},
\end{aligned}
$$




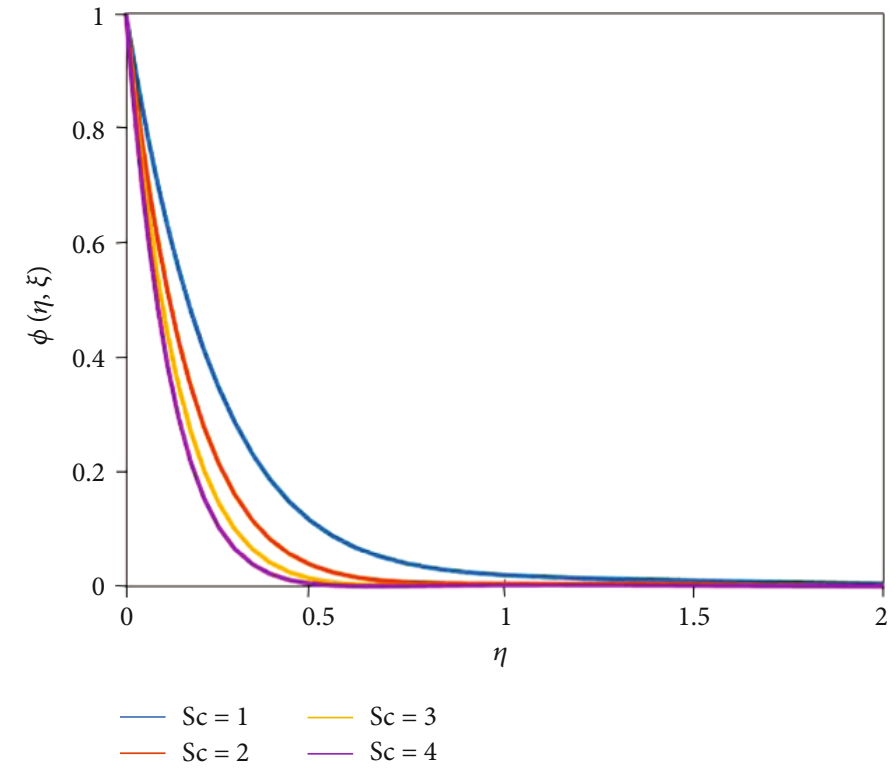

Figure 11: Concentration profile for different values of Sc when $\lambda=0.1, N=0.5, n=0.5, f_{w}=0.8, \xi=1, N_{b}=N_{t}=D f=B i=S r=0.5, \mathrm{He}$ $=\delta=N R=0.2, \mathrm{Sc}=0.6$, and $\operatorname{Pr}=1$.

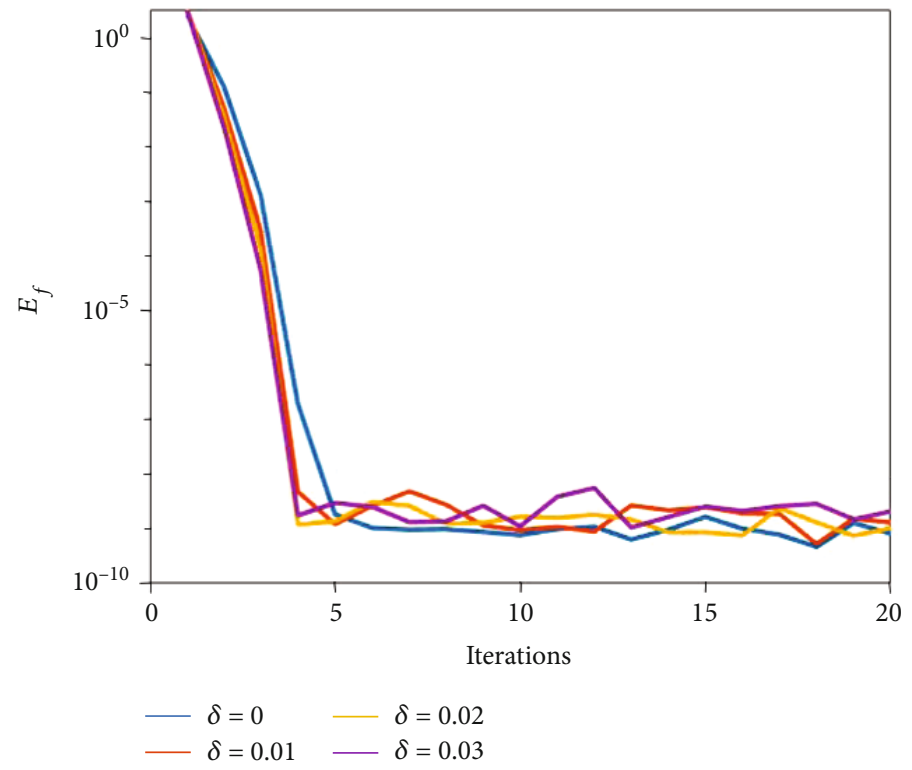

Figure 12: Concentration profile for different values of Pr when $D e=0.1, N=0.5, n=0.5, f_{w}=0.8, \xi=1, N_{b}=N_{t}=D f=S r=B i=0.5, \mathrm{He}$ $=\delta=N R=0.2, \mathrm{Sc}=0.6$, and $\operatorname{Pr}=1$.

where $\hat{d}_{j, q}=\left(\left(\xi_{m}-\xi_{m-1}\right) / 2\right) d_{j, q}, j, q=0,1,2, N_{t}$ are the entries of the differentiation matrix in the $m$ th subinterval. Substituting equations (32)-(37) into equations (22)-(24), we have

$$
\left[\mathbf{a}_{0, s}^{(m)} \mathbf{D}^{3}+\mathbf{a}_{1, s}^{(m)} \mathbf{D}^{2}+\mathbf{a}_{2, s}^{(m)} \mathbf{D}+\mathbf{a}_{3, s}^{(m)}\right] \mathbf{F}_{j, s+1}^{(m)}+\mathbf{a}_{4, s}^{(m)} \sum_{q=0}^{N_{t}} d_{j, q} \mathbf{D F}_{q, s+1}^{(m)}+\mathbf{a}_{5, s}^{(m)} \sum_{q=0}^{N_{t}} d_{j, q} \mathbf{F}_{q, s+1}^{(m)}+=\mathbf{R}_{1, j, s}^{(m)}
$$

$$
\begin{aligned}
& {\left[\mathbf{b}_{0, s}^{(m)} \mathbf{D}^{2}+\mathbf{b}_{1, s}^{(m)} \mathbf{D}+\mathbf{b}_{2, s}^{(m)}\right] \boldsymbol{\Theta}_{j, s+1}^{(m)}+\mathbf{b}_{3, s}^{(m)} \sum_{q=0}^{N_{t}} d_{j, q} \Theta_{q, s+1}^{(m)}} \\
& +\left[\mathbf{b}_{4, s}^{(m)} \mathbf{D}^{2}+\mathbf{b}_{5, s}^{(m)} \mathbf{D}+\mathbf{b}_{6, s}^{(m)}\right] \mathbf{F}_{j, s+1}^{(m)}+\mathbf{b}_{7, s}^{(m)} \sum_{q=0}^{N_{t}} d_{j, q} F_{q, s+1}^{(m)} \\
& +\left[\mathbf{b}_{8, s}^{(m)} \mathbf{D}\right] \boldsymbol{\Phi}_{j, s+1}^{(m)}=\mathbf{R}_{2, j, s}^{(m)},
\end{aligned}
$$




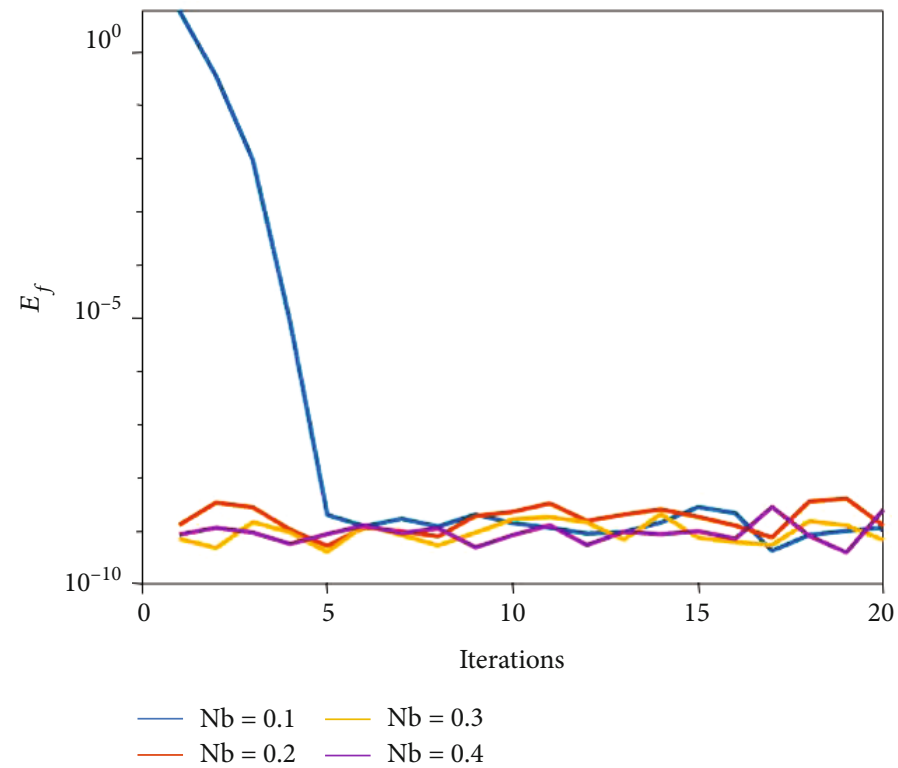

FIGURE 13: Concentration profile for different values of Sc when $\lambda=0.1, N=0.5, n=0.5, f_{w}=0.8, \xi=1, N_{b}=N_{t}=D f=B i=S r=0.5, \mathrm{He}$ $=\delta=N R=0.2, \mathrm{Sc}=0.6$, and $\operatorname{Pr}=1$.

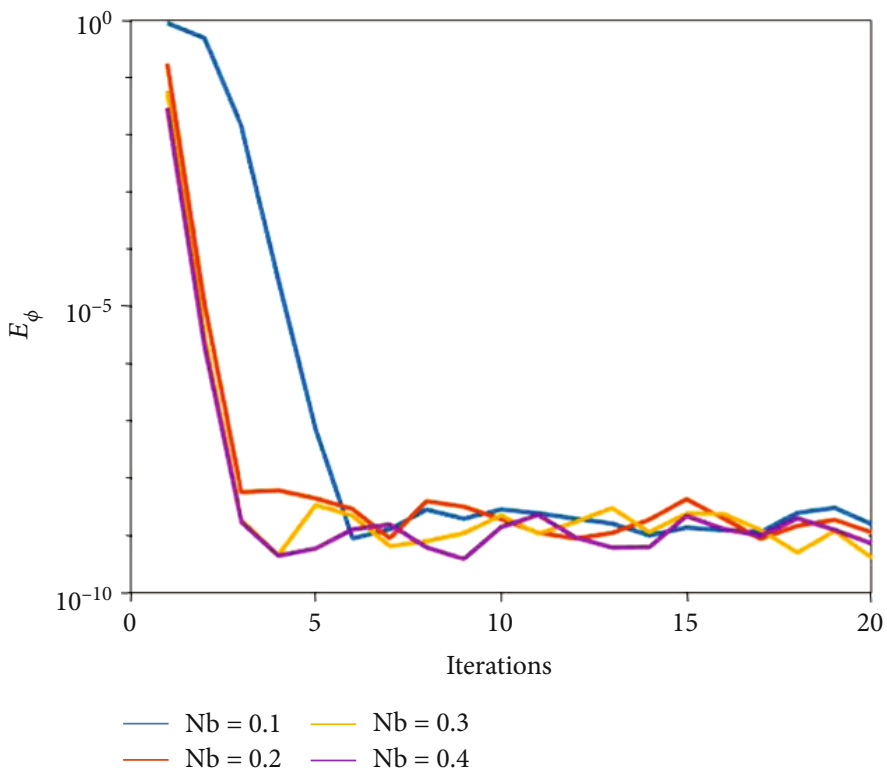

Figure 14: Concentration profile for different values of $\operatorname{Pr}$ when $D e=0.1, N=0.5, n=0.5, f_{w}=0.8, \xi=1, N_{b}=N_{t}=D f=S r=B i=0.5, \mathrm{He}$ $=\delta=N R=0.2, \mathrm{Sc}=0.6$, and $\operatorname{Pr}=1$.

$$
\begin{gathered}
{\left[\mathbf{c}_{0, s}^{(m)} \mathbf{D}^{2}+\mathbf{c}_{1, s}^{(m)} \mathbf{D}+\mathbf{c}_{2, s}^{(m)}\right] \boldsymbol{\Phi}_{j, s+1}^{(m)}+\mathbf{c}_{3, s}^{(m)} \sum_{q=0}^{N_{t}} d_{j, q} \boldsymbol{\Phi}_{q, s+1}^{(m)}} \\
+\left[\mathbf{c}_{4, r}^{(m)} \mathbf{D}+\mathbf{c}_{5, s}^{(m)}\right] \mathbf{F}_{j, r+1}^{(m)}+\mathbf{c}_{6, s}^{(m)} \sum_{q=0}^{N_{t}} d_{j, q} F_{q, s+1}^{(m)} \\
+\left[\mathbf{c}_{7, s}^{(m)} \mathbf{D}^{2}+\mathbf{c}_{8, s}^{(m)} \mathbf{D}\right] \boldsymbol{\Theta}_{j, s+1}^{(m)}=\mathbf{R}_{3, j, s}^{(m)}
\end{gathered}
$$

The solution at $j=N_{t}$ of each subinterval is obtained from the solution at the previous level, redenoting $i$ and $j$ indices; equations (42)-(44) can be written as

$$
\begin{aligned}
& {\left[\mathbf{a}_{0, s}^{(m)} \mathbf{D}^{3}+\mathbf{a}_{1, s}^{(m)} \mathbf{D}^{2}+\mathbf{a}_{2, s}^{(m)} \mathbf{D}+\mathbf{a}_{3, s}^{(m)}\right] \mathbf{F}_{i, s+1}^{(m)}+\mathbf{a}_{4, s}^{(m)} \sum_{j=0}^{N_{t}-1} d_{i, j} \mathbf{D} \mathbf{F}_{j, s+1}^{(m)}} \\
& \quad+\mathbf{a}_{5, s}^{(m)} \sum_{j=0}^{N_{t}-1} d_{i, j} \mathbf{F}_{j, s+1}^{(m)}=\mathbf{R}_{1, i, s}^{(m)}-\mathbf{a}_{4, s}^{(m)} d_{i, N_{t}} \mathbf{D} \mathbf{F}_{N_{t}, s+1}^{(m)} \\
& \quad-\mathbf{a}_{5, s}^{(m)} d_{i, N_{t}} \mathbf{F}_{N_{t}, s+1}^{(m)}
\end{aligned}
$$




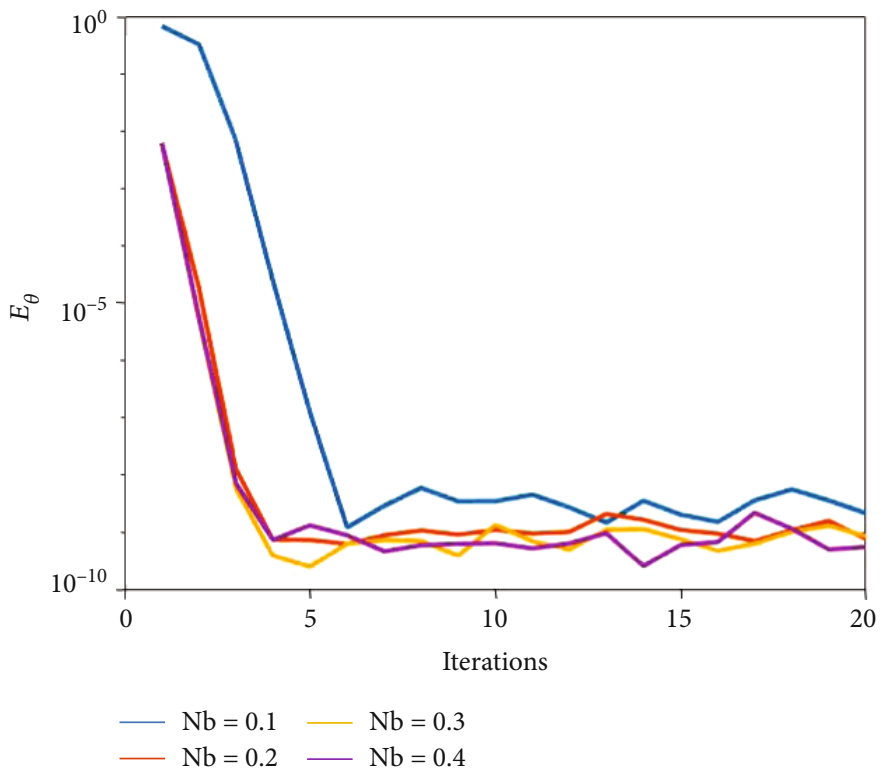

Figure 15: Concentration profile for different values of $S c$ when $\lambda=0.1, N=0.5, n=0.5, f_{w}=0.8, \xi=1, N_{b}=N_{t}=D f=B i=S r=0.5, \mathrm{He}$ $=\delta=N R=0.2, \mathrm{Sc}=0.6$, and $\operatorname{Pr}=1$.

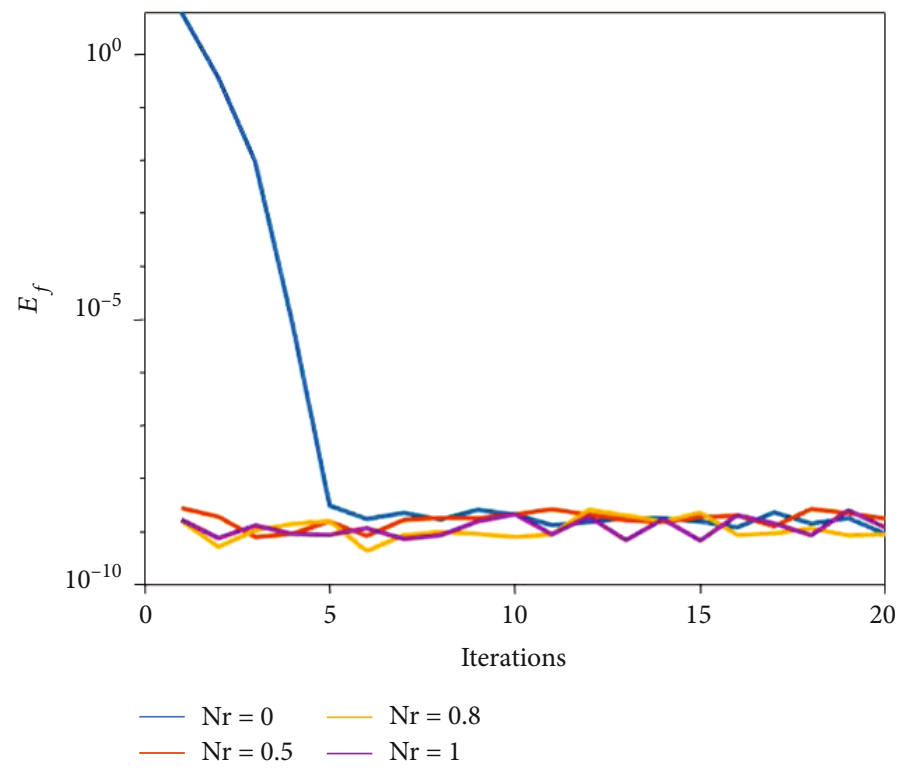

Figure 16: Concentration profile for different values of $\operatorname{Pr}$ when $D e=0.1, N=0.5, n=0.5, f_{w}=0.8, \xi=1, N_{b}=N_{t}=D f=S r=B i=0.5, \mathrm{He}$ $=\delta=N R=0.2, \mathrm{Sc}=0.6$, and $\operatorname{Pr}=1$.

$$
\begin{array}{ccc}
{\left[\mathbf{b}_{0, s}^{(m)} \mathbf{D}^{2}+\mathbf{b}_{1, s}^{(m)} \mathbf{D}+\mathbf{b}_{2, s}^{(m)}\right] \boldsymbol{\Theta}_{i, s+1}^{(m)}+\mathbf{b}_{3, s}^{(m)} \sum_{j=0}^{N_{t}-1} d_{i, j} \boldsymbol{\Theta}_{j, s+1}^{(m)}} & {\left[\mathbf{c}_{0, s}^{(m)} \mathbf{D}^{2}+\mathbf{c}_{1, s}^{(m)} \mathbf{D}+\mathbf{c}_{2, s}^{(m)}\right] \boldsymbol{\Phi}_{i, s+1}^{(m)}+\mathbf{c}_{3, s}^{(m)} \sum_{j=0}^{N_{t}-1} d_{i, j} \boldsymbol{\Phi}_{j, s+1}^{(m)}} \\
+\left[\mathbf{b}_{4, s}^{(m)} \mathbf{D}^{2}+\mathbf{b}_{5, s}^{(m)} \mathbf{D}+\mathbf{b}_{6, s}^{(m)}\right] \mathbf{F}_{i, s+1}^{(m)}+\mathbf{b}_{7, s}^{(m)} \sum_{j=0}^{N_{t}-1} d_{i, j} \mathbf{F}_{j, s+1}^{(m)} & +\left[\mathbf{c}_{4, s}^{(m)} \mathbf{D}+\mathbf{c}_{5, s}^{(m)}\right] \mathbf{F}_{i, s+1}^{(m)}+\mathbf{c}_{6, s}^{(m)} \sum_{j=0}^{N_{t}-1} d_{i, j} \mathbf{F}_{q, s+1}^{(m)} \\
+\left[b_{8, s}^{(m)}\right] \boldsymbol{\Phi}_{i, s+1}^{(m)}=\mathbf{R}_{2, i, s}^{(m)}-\mathbf{b}_{3, s}^{(m)} d_{i, N_{t}} \boldsymbol{\Theta}_{N_{t}, s+1}^{(m)}-\mathbf{b}_{7, s}^{(m)} d_{i, N_{t}} \mathbf{F}_{N_{t}, s+1}^{(m)} & +\left[\mathbf{c}_{7, s}^{(m)} \mathbf{D}^{2}+\mathbf{c}_{8, s}^{(m)} \mathbf{D}\right] \boldsymbol{\Theta}_{i, s+1}^{(m)} \\
& =\mathbf{R}_{3, i, s}^{(m)}-\mathbf{c}_{3, s}^{(m)} d_{i, N_{t}} \boldsymbol{\Phi}_{N_{t}, s+1}^{(m)}-\mathbf{c}_{6, s}^{(m)} d_{i, N_{t}} \mathbf{F}_{N_{t}, s+1}^{(m)}
\end{array}
$$




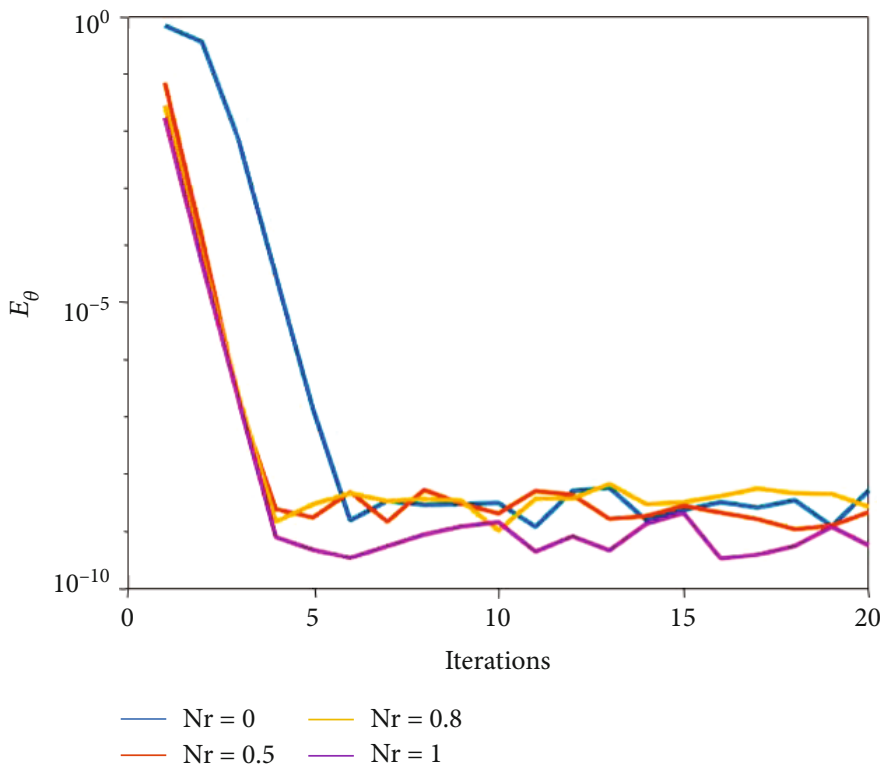

Figure 17: Concentration profile for different values of Sc when $\lambda=0.1, N=0.5, n=0.5, f_{w}=0.8, \xi=1, N_{b}=N_{t}=D f=B i=S r=0.5, \mathrm{He}$ $=\delta=N R=0.2, \mathrm{Sc}=0.6$, and $\operatorname{Pr}=1$.

For brevity, equations (45)-(47) can be written as

$$
\begin{aligned}
& \mathbf{A}_{1,1}^{(i)} \mathbf{F}_{i, s+1}^{(m)}+\mathbf{a}_{4, s}^{(m)} \sum_{j=0}^{N_{t}-1} d_{i, j} \mathbf{D} \mathbf{F}_{j, s+1}^{(m)}+\mathbf{a}_{5, s}^{(m)} \sum_{j=0}^{N_{t}-1} d_{i, j} \mathbf{F}_{j, s+1}^{(m)}+\mathbf{A}_{1,2}^{(i)} \boldsymbol{\Theta}_{i, s+1}^{(m)} \\
& +\mathbf{A}_{1,3}^{(i)} \boldsymbol{\Phi}_{i, s+1}^{(m)}=\boldsymbol{\beta}_{1, i, s}^{(m)} \\
& \mathbf{A}_{2,1}^{(i)} \mathbf{F}_{i, s+1}^{(m)}+\mathbf{b}_{7, s}^{(m)} \sum_{j=0}^{N_{t}-1} d_{i, j} \mathbf{F}_{j, s+1}^{(m)}+\mathbf{A}_{2,2}^{(i)} \boldsymbol{\Theta}_{i, s+1}^{(m)}+\mathbf{b}_{3, s}^{(m)} \sum_{j=0}^{N_{t}-1} d_{i, j} \boldsymbol{\Theta}_{j, s+1}^{(m)} \\
& +\mathbf{A}_{2,3}^{(i)} \boldsymbol{\Phi}_{i, s+1}^{(m)}=\boldsymbol{\beta}_{2, i, s}^{(m)} \\
& \mathbf{A}_{3,1}^{(i)} \mathbf{F}_{i, s+1}^{(m)}+\mathbf{c}_{6, s}^{(m)} \sum_{j=0}^{N_{t}-1} d_{i, j} \mathbf{F}_{j, s+1}^{(m)}+\mathbf{A}_{3,2}^{(i)} \boldsymbol{\Theta}_{i, s+1}^{(m)}+\mathbf{A}_{3,3}^{(i)} \boldsymbol{\Phi}_{i, s+1}^{(m)} \\
& +\mathbf{c}_{3, s}^{(m)} \sum_{j=0}^{N_{t}-1} d_{i, j} \boldsymbol{\Phi}_{j, s+1}^{(m)}=\boldsymbol{\beta}_{3, i, s}^{(m)}
\end{aligned}
$$

where

$$
\begin{gathered}
\mathbf{A}_{1,1}^{(i)}=\mathbf{a}_{0, s}^{(m)} \mathbf{D}^{3}+\mathbf{a}_{1, s}^{(m)} \mathbf{D}^{2}+\mathbf{a}_{2, s}^{(m)} \mathbf{D}+\mathbf{a}_{3, s}^{(m)}, \\
\mathbf{A}_{1,2}^{(i)}=\mathbf{0}, \\
\mathbf{A}_{1,3}^{(i)}=\mathbf{0}, \\
\mathbf{A}_{2,1}^{(i)}=\mathbf{b}_{4, s}^{(m)} \mathbf{D}^{2}+\mathbf{b}_{5, s}^{(m)} \mathbf{D}+\mathbf{b}_{6, s}^{(m)}, \\
\mathbf{A}_{2,2}^{(i)}=\mathbf{b}_{0, s}^{(m)} \mathbf{D}^{2}+\mathbf{b}_{1, s}^{(m)} \mathbf{D},+\mathbf{b}_{2, s}^{(m)}, \\
\mathbf{A}_{2,3}^{(i)}=b_{8, s}^{(m)} \\
\mathbf{A}_{3,1}^{(i)}=\mathbf{c}_{4, s}^{(m)} \mathbf{D}+\mathbf{c}_{5, s}^{(m)},
\end{gathered}
$$

$$
\begin{gathered}
\mathbf{A}_{3,2}^{(i)}=\mathbf{c}_{7, s}^{(m)} \mathbf{D}^{2}+\mathbf{c}_{8, s}^{(m)} \mathbf{D}, \\
\mathbf{A}_{3,3}^{(i)}=\mathbf{c}_{0, s}^{(m)} \mathbf{D}^{2}+\mathbf{c}_{1, s}^{(m)} \mathbf{D},+\mathbf{c}_{2, s}^{(m)} \\
\boldsymbol{\beta}_{1, i, s}^{(m)}=\mathbf{R}_{1, i, s}^{(m)}-\mathbf{a}_{4, s}^{(m)} d_{i, N_{t}} \mathbf{D} \mathbf{F}_{N_{t}, s s+1}^{(m)}-\mathbf{a}_{5, s}^{(m)} d_{i, N_{t}} \mathbf{F}_{N_{t}, s+1}^{(m)}, \\
\boldsymbol{\beta}_{2, i, s}^{(m)}=\mathbf{R}_{2, i, s}^{(m)}-\mathbf{b}_{3, s}^{(m)} d_{i, N_{t}} \boldsymbol{\Theta}_{N_{t}, s+1}^{(m)}-\mathbf{b}_{7, s}^{(m)} d_{i, N_{t}} \mathbf{F}_{N_{t}, s+1}^{(m)}, \\
\boldsymbol{\beta}_{3, i, s}^{(m)}=\mathbf{R}_{3, i, s}^{(m)}-\mathbf{c}_{3, s}^{(m)} d_{i, N_{t}} \boldsymbol{\Phi}_{N_{t}, s+1}^{(m)}-\mathbf{c}_{6, s}^{(m)} d_{i, N_{t}} \mathbf{F}_{N_{t}, s+1}^{(m)} .
\end{gathered}
$$

\section{Results and Discussion}

A numerical solution for the boundary layer nanofluid flow along a moving cylinder with thermal radiation, heat generation, and slip effects is studied. The solutions for the system of partial differential equations are presented. The bivariate spectral quasilinearization method (MD-BSQLM) is used to solve the coupled system. To validate the numerical method, a comparison with results in [36] was made, and the results are in acceptable agreement. The finite difference-based method used in [36] shows a slightly more rigorous approach than the one applied in this study.

We present important and interesting results only. The results obtained are presented in Table 1 and Figures 2-18. The discussion of the effect of the Prandtl number and the magnetic parameter is solved for the purpose of validating results obtained in the literature as they have been widely reported. The most interesting and important results include the effect of the Brownian motion $N_{b}$, the thermophoresis parameter $N_{t}$, heat generation $\mathrm{He}$, velocity slip parameter $\delta$, the temperature exponent $m$, and the radial coordinate $r$ . The values $M=0, N_{r}=m=\mathrm{Ec}=N_{b}=N_{t}=\mathrm{He}=r=\delta=0$, 


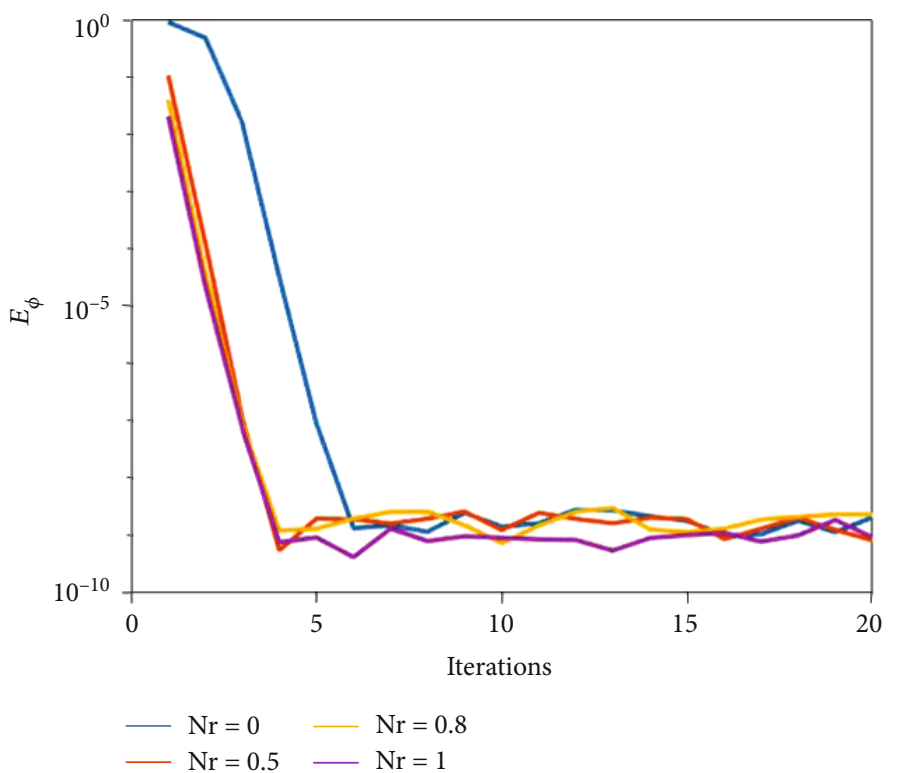

Figure 18: Concentration profile for different values of $\operatorname{Pr}$ when $D e=0.1, N=0.5, n=0.5, f_{w}=0.8, \xi=1, N_{b}=N_{t}=D f=S r=B i=0.5, \mathrm{He}$ $=\delta=N R=0.2, \mathrm{Sc}=0.6$, and $\operatorname{Pr}=1$.

and $\partial f / \partial \xi=\partial f^{\prime} / \partial \xi=0$ give the set of equations (13)-(15) to those of [36].

Figures 2 and 3 show the effect of varying the velocity slip parameter $\delta$ on the velocity and temperature profiles. Increasing $\delta$ result in increasing both the velocity and temperature profiles. Increasing the velocity slip parameter causes the fluid layer in contact with the cylinder surface to slip thereby causing the velocity at the surface to increase. The velocity decrease with increasing boundary layer. When fluid particles move faster on the cylinder surface, this causes more fluid particle interaction causing the temperature to increase with increasing $\delta$.

Figures 4 and 5 depict the effect of changing the magnetic parameter $M$ on velocity profiles and changing the heat generation parameter on the temperature profiles. Increasing the magnetic parameter result in the decrease in velocity profiles, this result agrees with many other results displayed in the literature in $[20,37]$ and [21]. If the heat generation parameter is increased, this then results in the increase in the temperature profiles; this result has been shown in many other different studies. This shows a strong agreement in the results when the MD-BSQLM is used.

Figure 6 shows the effect of increasing the radiation parameter $N_{r}$ on the temperature profiles. If the radiation parameter is increased, this has an effect of increasing temperature profiles. A point of "inflexion" is noticed close to the surface of the cylinder. When radiation is applied, it causes a rise in temperature causing the mass particles close to the surface with lower temperature to rise. They then become heated as they move further from the surface causing the temperature to increase further away from this point.

Figures 7 and 8 show the effect of varying the Eckert number Ec and the radial coordinate $r$. Increasing the Eckert number results in the increase in temperature profiles. The trends in the increase in temperature fields are more pro- nounced at close to the cylinder surface. This is caused by the presence of velocity slip and radiation which increase temperature at the surface. The gradient of the velocity profile at the surface $-\theta^{\prime}(0)$ (heat transfer coefficient) can be seen increasing from negative to positive. Increasing the radial coordinate shows a decrease in the concentration profiles. This shows that as we move outward from the cylinder surface, the mass particle concentration decreases. This is consistent with the general settling of mass particles to the cylinder surface due to gravity. The settling is less enhanced due to the fluid flow which tends to carry the particles along with the flow regime.

Figures 9 and 10 show the effect of varying the thermophoresis parameter $N_{t}$ and the the Brownian motion parameter $N_{b}$ and on the concentration profiles. If the Brownian motion parameter is increased, this results in decreasing the concentration profile at close to the surface. A reverse effect is noticed further away from the cylinder surface. Increasing the Brownian motion parameter causes the mass particles to move more rapidly in random motion this destabilizes the particle motion. A more enhanced point of inflexion is noticed. Increasing the thermophoresis parameter $N_{t}$ results in decreasing the concentration profiles at close to the cylinder surface.

In Figure 11, it is shown that if the Schmidt number Sc is increased, this result in the decrease in the concentration profiles. The result is consistent with those in the literature.

The effect of changing parameters of the coupled partial differential equations affects the stability of the numerical solution. Sometimes when parameters exceed certain ranges, the numerical method fails. In this section, we study the effect of changing various parameters on the stability of the numerical solution. This is shown in the graphs from Figures $12-18$. The effect of varying the velocity slip $\delta$ on the residual error of the solution of $f$, the Brownian motion 
$N_{b}$ on the residual error of $f, \theta$, and $\phi$, and $N_{r}$ on $f, \theta$, and $\phi$. Figure 12 shows the effect of increasing the velocity slip $\delta$ on the residual error of the solution of $f$; the error does not deteriorate with increasing iterations. The sudden drop in residual error in less than five iterations shows the accuracy of the MD BSQLM. Figure 13 shows the effect of increasing the Brownian motion parameter $N_{b}$ on the residual error of $f$; the residual error becomes very small with larger values of the Brownian motion parameter. The error becomes more stable with increasing iterations. In Figures 14 and 15, the errors $E_{\phi}$ and $E_{\theta}$ improve with increasing Brownian motion parameter. At low values of the parameter convergence is delayed, happens after five iterations. In both cases, the error does not deteriorate with increasing iterations.

In Figures 16-18, it is shown that increasing the radiation parameter does not make the errors $E_{f}, E_{\phi}$, and $E_{\theta}$ deteriorate with increasing iterations. Increasing the radiation parameter $N_{r}$ result in improving the stability of the errors of the MD-BSQLM.

\section{Conclusion}

In this paper, we have studied the numerical solution of laminar boundary layer flow along with a moving cylinder with thermal radiation, heat generation, and surface slip effect. The coupled partial differential equations of the mass and heat transfer along with a cylinder in motion are solved by the multidomain bivariate spectral quasilinearization method (MD BSQLM). The most important results show that increasing the velocity slip factor results in an enhanced increase in velocity and temperature profiles. Increasing the heat generation parameter increases temperature profiles; if the radiation parameter and the Eckert number are both increased, this would then result in the increase temperature trends. The concentration fields decrease with increasing radial coordinate. Increasing the thermophoresis parameter and Brownian motion both destabilizes the concentration profiles. Increasing the Schmidt number reduce temperature fields. The effect of increasing selected parameters: the velocity slip, Brownian motion, and radiation parameter on all residual errors show that these errors do not deteriorate. This shows that the multidomain bivariate quasilinearization method (MD BSQLM) is very accurate and robust. The method is also easier to implement than the traditional finite difference methods as shown in Section 3. The method also shows very low residual errors only after five iterations. The method adds value to the way in which differential equations arising from fluid dynamics are solved.

\section{Nomenclature}

$u$ : $\quad$ Velocity component in $x$ direction $\left(\mathrm{ms}^{-1}\right)$

$v$ : Velocity component in $y$ direction $\left(\mathrm{ms}^{-1}\right)$

$C_{\infty}$ : Solute ambient concentration $\left(\mathrm{kgm}^{3}\right)$

$C_{w}$ : Surface solute concentration $\left(\mathrm{kgm}^{3}\right)$

$T_{\infty}$ : Fluid ambient temperature $\left({ }^{\circ} \mathrm{C}\right)$

$T_{w}$ : Fluid temperature at cylinder surface $\left({ }^{\circ} \mathrm{C}\right)$

$r$ : Radial coordinate $v: \quad$ Kinematic viscosity $\left(\mathrm{m}^{2} \mathrm{~s}^{-1}\right)$

$\sigma: \quad$ Electrical conductivity $\left(\mathrm{Sm}^{-1}\right)$

$\rho: \quad$ Fluid density $\left(\mathrm{kgm}^{-3}\right)$

$B_{0}$ : $\quad$ Magnetic field $(T)$

$C_{p}:$ Specific heat capacity $\left(\mathrm{J} / \mathrm{kg}^{\circ} \mathrm{C}\right.$

$\sigma^{*}:$ Boltzmann constant $\left(\mathrm{JK}^{-1}\right)$

$k^{*}$ : Mean absorption coefficient $\left(\mathrm{m}^{2} \mathrm{~mol}^{-1}\right)$

$k$ : Thermal diffusivity $\left(\mathrm{m}^{2} \mathrm{~s}^{-1}\right)$

$\mu$ : $\quad$ Dynamic viscosity (Pas)

$\tau: \quad$ Nondimensional ratio of specific heat capacities

$D_{B}$ : Brownian diffusion coefficient

$D_{T}$ : Thermophoretic diffusion coefficient $\left(\mathrm{m}^{2} \mathrm{~mol}^{-1}\right)$

$Q_{0}:$ Heat generation constant $\left(\mathrm{ms}^{-1}\right)$

$R: \quad$ Radius of cylinder (m)

$B_{1}$ : Velocity slip factor $\left(\mathrm{ms}^{-1}\right)$

$U_{\infty}$ : Free stream velocity $\left(\mathrm{ms}^{-1}\right)$

$U_{w}:$ Surface velocity $\left(\mathrm{ms}^{-1}\right)$

$M$ : $\quad$ Magnetic parameter $\left(\mathrm{ms}^{-1}\right)$

$N_{r}:$ Thermal radiation parameter $\left(\mathrm{ms}^{-1}\right)$

Pr: Prandtl number

$m$ : Temperature exponent

Ec: Eckert number

$N_{b}$ : Brownian motion parameter

$N_{t}$ : Thermophoresis parameter

He: Nondimensinal heat generation parameter

Sc: Schmidt number

$\delta$ : Nondimensional velocity slip parameter

\section{Data Availability}

No data was used in this study; calculations were performed from code simulations in Matlab.

\section{Conflicts of Interest}

The authors declare that there is no conflict of interest regarding the publication of this article.

\section{Acknowledgments}

This work is supported by the National Research Foundation (NRF) of South Africa (Grant Number 129490), the DSI-NRF Centre of Excellence in Mathematical and Statistical Sciences (CoE-MaSS), South Africa, and the Central University of Technology, South Africa.

\section{References}

[1] V. Poply, P. Singh, and K. K. Chaudhary, "Analysis of laminar boundary layer flow along a stretching cylinder in the presence of thermal radiation," WSEAS Transactions on Fluid Mechanics, vol. 8, no. 4, pp. 159-164, 2013.

[2] S. Shateyi and G. T. Marewo, "A new numerical approach for the laminar boundary layer flow and heat transfer along a stretching cylinder embedded in a porous medium with variable thermal conductivity," Journal of Applied Mathematics, vol. 2013, Article ID 576453, 7 pages, 2013.

[3] R. R. Rangi and N. Ahmad, "Boundary layer flow past a stretching cylinder and heat transfer with variable thermal 
conductivity," Applied Mathematics, vol. 3, no. 3, pp. 205-209, 2012.

[4] S. Mukhopadhyay, "Analysis of boundary layer flow and heat transfer along a stretching cylinder in a porous medium," International Scholarly Research Network, vol. 2012, article 704984, pp. 1-7, 2012.

[5] E. M. A. Elbashbeshy, T. G. Emam, M. S. El-Azab, and K. M. Abdelgaber, "Laminar boundary layer flow along a stretching cylinder embedded in a porous medium," International Journal of the Physical Sciences, vol. 7, no. 24, pp. 3067-3072, 2012.

[6] H. T. Lin and Y. P. Shih, "Laminar boundary layer heat transfer along a statitic and moving cylinders," Journal of the Chinese Institute of Engineers, vol. 3, no. 1, pp. 73-79, 1980.

[7] M. Ali and A. Alabdulkarem, "Laminar mixed convection boundary layer flow induced by a permeable surface stretched with prescribed skin friction boundary conditions," Advances in Mechanical Engineering, vol. 7, no. 9, 2015.

[8] S. U. S. Choi, Enhancing thermal conductivity of fluids with nanoparticles, vol. 231, ASME-Publications-Federation, 1995.

[9] J. Buongiorno, "Convective transport in nanofluids," Journal of Heat Transfer, vol. 128, no. 3, pp. 240-250, 2006.

[10] S. Z. Alamri, R. Ellahi, N. Shehzad, and A. Zeeshan, "Convective radiative plane Poiseuille flow of nanofluid through porous medium with slip: an application of Stefan blowing," Journal of Molecular Liquids, vol. 273, pp. 292-304, 2019.

[11] S. Nadeem, R. Mehmood, and N. S. Akbar, "Oblique stagnation point flow of a Casson-nano fluid towards a stretching surface with heat transfer," Journal of Computational and Theoretical Nanoscience, vol. 11, no. 6, pp. 1422-1432, 2014.

[12] M. Khan, M. Y. Malik, T. Salahuddin, and F. Khan, "Generalized diffusion effects on Maxwell nanofluid stagnation point flow over a stretchable sheet with slip conditions and chemical reaction," Journal of the Brazilian Society of Mechanical Sciences and Engineering, vol. 41, no. 3, p. 138, 2019.

[13] R. Dhanai, P. Rana, and L. Kumar, "Multiple solutions of MHD boundary layer flow and heat transfer behavior of nanofluids induced by a power-law stretching/shrinking permeable sheet with viscous dissipation," Powder Technology, vol. 273, pp. 62-70, 2015.

[14] S. Shah, S. Islam, H. Ayaz, and S. Khan, "Radiative heat and mass transfer analysis of micropolar nanofluid flow of Casson fluid between two rotating parallel plates with effects of Hall current," Journal of Heat Transfer, vol. 141, no. 2, pp. 022401-022413, 2019.

[15] S. Nadeem, M. Riaz khan, and A. U. Khan, "MHD oblique stagnation point flow of nanofluid over an oscillatory stretching/shrinking sheet: existence of dual solutions," Physica Scripta, vol. 94, no. 7, article 075204, 2019.

[16] F. Kamal, K. Zaimi, A. Ishak, and I. Pop, "Stability analysis of MHD stagnation-point flow towards a permeable stretching/ shrinking sheet in a nanofluid with chemical reactions effect," Sains Malaysiana, vol. 48, no. 1, pp. 243-250, 2019.

[17] P. Besthapu, R. U. Haq, S. Bandari, and Q. M. Al-Mdallal, "Thermal radiation and slip effects on MHD stagnation point flow of non-Newtonian nanofluid over a convective stretching surface," Neural Computing and Applications, vol. 31, no. 1, pp. 207-217, 2019.

[18] M. A. Sadiq, A. U. Khan, S. Saleem, and S. Nadeem, "Numerical simulation of oscillatory oblique stagnation point flow of a magneto micropolar nanofluid," RSC Advances, vol. 9, no. 9, pp. 4751-4764, 2019.
[19] M. K. Nayak, T. M. Agbaje, S. Mondal, P. Sibanda, and P. G. L. Leach, "Thermodynamic effect in Darchy-Forchheimer nanofluid flow of a single-wall carbon nanotube/multi-wall carbon nanotube suspension due to a stretching/shrinking rotating disk: Buongiorno two-phase model," Journal of Engineering Mathematics, vol. 120, no. 1, pp. 43-65, 2020.

[20] M. A. Yousif, H. F. Ismael, T. Abbas, and R. Ellahi, "Numerical study of momentum and heat transfer of MHD Carreau nanofluid over an exponentially stretched plate with internal heat source/sink and radiation," Heat Transfer Research, vol. 50, no. 7, pp. 649-658, 2019.

[21] S. O. Salawu and H. A. Ogunseye, "Entropy generation of a radiative hydromagnetic Powell-Eyring chemical reaction nanofluid with variable conductivity and electric field loading," Results in Engineering, vol. 5, article 100072, 2020.

[22] B. Kumar and S. Srinivas, "Unsteady hydromagnetic flow of Eyring-Powell nanofluid over an inclined permeable stretching sheet with joule heating and thermal radiation," Journal of Applied and Computational Mechanics, vol. 6, no. 2, pp. 259-270, 2020.

[23] P. Sreedevi and P. S. Reddy, "Effect of SWCNTs and MWCNTs Maxwell MHD nanofluid flow between two stretchable rotating disks under convective boundary conditions," Heat Transfer-Asian Research, vol. 48, no. 8, pp. 4105-4132, 2019.

[24] P. Sreedevi, P. S. Reddy, and M. Sheremet, "A comparative study of Al2O3and $\mathrm{TiO} 2$ nanofluid flow over a wedge with nonlinear thermal radiation," International Journal of Numerical Methods for Heat \& Fluid Flow, vol. 30, no. 3, pp. 12911317, 2019.

[25] B. J. Gireesha, M. Archana, B. Mahanthesh, and B. C. Prasannakumara, "Exploration of activation energy and binary chemical reaction effects on nano Casson fluid flow with thermal and exponential space-based heat source," Multidiscipline Modeling in Materials and Structures, vol. 15, no. 1, pp. 227245, 2019.

[26] B. J. Gireesha, M. Umeshaiah, B. C. Prasannakumara, N. S. Shashikumar, and M. Archana, "Impact of nonlinear thermal radiation on magnetohydrodynamic three dimensional boundary layer flow of Jeffrey nanofluid over a nonlinearly permeable stretching sheet," Physica A: Statistical Mechanics and its Applications, vol. 549, article 124051, 2020.

[27] M. Archana, B. J. Gireesha, and B. C. Prasannakumara, "Triple diffusive flow of Casson nanofluid with buoyancy forces and nonlinear thermal radiation over a horizontal plate," Archives of Thermodynamics, vol. 40, no. 1, pp. 49-69, 2019.

[28] M. Awais, S. Bilal, M. Y. Malik, and Khalil-ur-Rehman, "Numerical analysis of magnetohydrodynamic Navier's slip visco nanofluid flow induced by rotating disk with heat source/sink," Communications in Theoretical Physics, vol. 71, no. 9, p. 1075, 2019.

[29] R. E. Bellman and R. E. Kalaba, Quasilinearization and nonlinear boundary-value problems, Elsevier Publishing Company, New York, 1965.

[30] S. S. Motsa, "On the bivariate spectral homotopy analysis method approach for solving nonlinear evolution partial differential equations," Abstract and Applied Analysis, vol. 2014, Article ID 350529, 8 pages, 2014.

[31] S. S. Motsa, V. M. Magagula, and P. Sibanda, "A bivariate Chebyshev spectral collocation quasilinearization method for nonlinear evolution parabolic equations," The Scientific World Journal, vol. 2014, no. 13, Article ID 581987, 13 pages, 2014. 
[32] V. M. Magagula, S. S. Motsa, P. Sibanda, and P. G. Dlamini, "On a bivariate spectral relaxation method for unsteady magneto-hydrodynamic flow in porous media," Springerplus, vol. 5, no. 1, pp. 1-15, 2016.

[33] S. S. Motsa, P. G. Dlamini, and M. Khumalo, "Spectral relaxation method and spectral quasilinearization method for solving unsteady boundary layer flow problems," Advances in Mathematical Physics, vol. 2014, Article ID 341964, 12 pages, 2014.

[34] L. N. Trefethen, Spectral Methods in MATLAB, SIAM, Philadelphia, 2000.

[35] C. Canuto, M. Y. Hussaini, A. Quarteroni, and T. A. Zang, Spectral Methods in Fluid Dynamics, Springer-Verlag, Berlin, 1988.

[36] A. Ishak, R. Nazar, and I. Pop, "Local similarity solutions for laminar boundary layer flow along a moving cylinder in a parallel stream," in Computer Mathematics. ASCM 2007, D. Kapur, Ed., vol. 5081 of Lecture Notes in Computer Science, Springer, Berlin, Heidelberg, 2008.

[37] G. S. Seth, B. Kumar, and R. Nandkeolyar, "MHD mixed convection stagnation point flow of a micropolar nanofluid adjacent to stretching sheet: a revised model with successive linearization method," Journal of Nanofluids, vol. 8, no. 3, pp. 620-630, 2019. 